\title{
Assessment of Unabated Facility Emission Potentials for Evaluating Airborne Radionuclide Monitoring Requirements at Pacific Northwest National Laboratory - 1999
}

September 1999

Prepared for

the U.S. Department of Energy under Contract DE-AC06-76RLO 1830

Pacific Northwest National Laboratory

Richland, Washington 99352 



\section{DISCLAIMER}

This report was prepared as an account of work sponsored by an agency of the United States Government. Neither the United States Government nor any agency thereof, nor any of their employees, make any warranty, express or implied, or assumes any legal liability or responsibility for the accuracy, completeness, or usefulness of any information, apparatus, product, or process disclosed, or represents that its use would not infringe privately owned rights. Reference herein to any specific commercial product, process, or service by trade name, trademark, manufacturer, or otherwise does not necessarily constitute or imply its endorsement, recommendation, or favoring by the United States Government or any agency thereof. The views and opinions of authors expressed herein do not necessarily state or reflect those of the United States Government or any agency thereof. 


\section{DISCLAIMER}

Portions of this document may be illegible in electronic image products. Images are produced from the best available original document. 


\section{Summary}

Assessments were performed to evaluate compliance with the airborne radionuclide emission monitoring requirements in the National Emission Standards for Hazardous Air Pollutants (NESHAP - U.S. Code of Federal Regulations, Title 40 Part 61, Subpart H). In these assessments, potential unabated offsite doses were evaluated for emission locations at facilities owned by the U.S. Department of Energy and operated by Pacific Northwest National Laboratory (Pacific Northwest) on the Hanford Site. Two of the facilities evaluated, 325 Building Radiochemical Processing Laboratory, and 331 Building Life Sciences Laboratory met state and federal criteria for continuous sampling of airborne radionuclide emissions. One other building, the 3720 Environmental Sciences Laboratory, was recognized as being in transition with the potential for meeting the continuous sampling criteria.

The assessments were performed using building radionuclide inventory data obtained in 1999. The list of buildings evaluated is provided in Table S.1.

Table S.1. PNNL-Operated DOE Buildings with Radioactive Inventories in 1999.

200 Environmental Monitoring Shed

2718E Critical Mass Fissile Storage 300-N Caisson

305-B Hazardous Waste Storage Facility

306-W Materials Development Building

314 Engineering Development Laboratory

318 Radiological Calibrations Laboratory

320 Analytical and Nuclear Research

Laboratory

323 Mechanical Properties Laboratory

325 Radiochemical Processing Laboratory

326 Materials Sciences Laboratory

329 Chemical Sciences Laboratory

331 Life Sciences Laboratory
331-G Interim Tissue Repository

331-H Aerosol Wind Tunnel Research Facility

3718A Lab Equipment Central Pool

3720 Environmental Sciences Laboratory

3730 Gamma Irradiation Facility

3745 Radiological Calibrations \& Standards

Building

3020 Environmental Molecular Sciences

Laboratory

622-R Meteorological Laboratory

6652 Arid Land Ecology Laboratory

747A Whole Body Counter

Field Lysimeter Test Facility (FLTF)

Prototype Surface Barrier (PSB) 


\section{Contents}

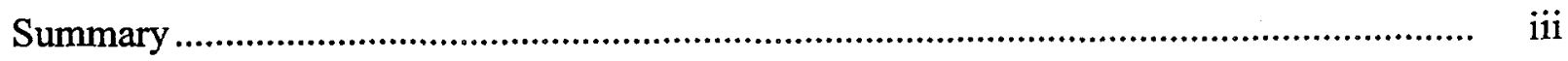

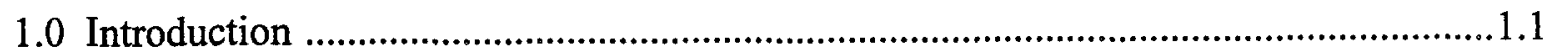

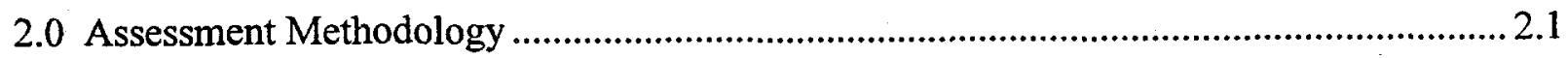

2.1 Projections of Annual Emission Quantities............................................................... 2.1

2.2 Maximum Receptor Unit Dose Calculation .............................................................. 2.4

2.3 Potential Emission Dose Assessment ...................................................................... 2.5

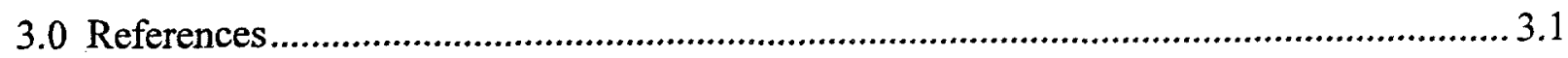

Appendix A - Common Radionuclide Mixtures ........................................................................

Appendix B - Radionuclide Inventory Database Features........................................................... B.1

Appendix C - Unit Dose Factor Calculations ........................................................................

Tables

S.1 PNNL-Operated DOE Buildings with Radioactive Inventories in 1999 .......................... iii

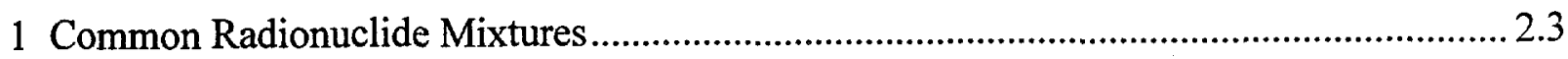

2 Physical Forms and Potential Annual Release Fractions for Radionuclides ......................... 2.4

3 Unit Dose Factors for 300 Area Source Location............................................................. 2.6

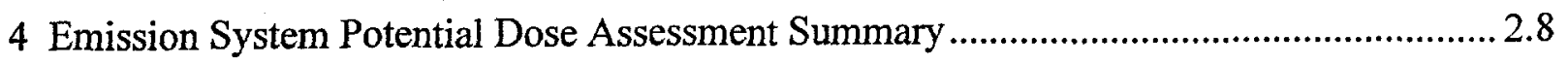




\subsection{Introduction}

Requirements for sampling for airborne radionuclide emissions are contained in the following regulations and guides:

- U.S. Code of Federal Regulations (CFR), Title 40, Subpart H: National Emission Standards for Hazardous Air Pollutants (NESHAP)(1989)

- Washington Administrative Code (WAC) 246-247: Radiation Protection - Air Emissions (1994)

- U.S. Department of Energy, DOE 5400.1: General Environmental Protection Program (1990)

- U.S. Department of Energy, DOE/EH-0173T, Environmental Regulatory Guide for Radiological Effluent Monitoring and Environmental Surveillance (January 1991).

These documents require that continuous sampling be performed at emission points that have the potential to cause an offsite dose of $0.1 \mathrm{mrem} / \mathrm{yr}$ if routine emissions were not mitigated by engineered pollution control systems. In addition, DOE 5400.1 specifies that a written plan be prepared for each facility that uses, generates, releases, or manages significant pollutants or hazardous materials. Thus, a Facility Effluent Monitoring Plan (FEMP) is prepared for facilities that require continuous sampling according to NESHAP.

In response to these requirements, the potential unmitigated offsite receptor dose from the facilities operated by the U.S. Department of Energy's (DOE's) Pacific Northwest National Laboratory $^{\text {(a) }}$ (Pacific Northwest) containing radioactive materials or sources is evaluated annually. These evaluations were performed initially in 1991 for DOE's Pacific Northwest facilities on the Hanford Site. Based on the initial assessments, four Pacific Northwest buildings were identified as containing a sufficient inventory of radioactive material such that unmitigated emissions could potentially result in an annual offsite maximum receptor dose of $0.1 \mathrm{mrem}$. These buildings were the 324 Waste Technology Engineering Laboratory, the 325 Applied Chemistry Laboratory, the 327 Postirradiation Testing Laboratory, and the 3720 Chemistry and Material Sciences Laboratory. In accordance with the NESHAP, qualifying emission points from these buildings were sampled continuously. Also, in accordance with DOE 5400.1, FEMPs were prepared for these buildings. ${ }^{(b)}$

The original radionuclide assessments were updated annually. There were no changes in the facility emission NESHAP status until 1996 when the 324 and 327 Buildings were transitioned to Babcock \&Wilcox. In 1997 , proposed movement of the radionuclide inventory from the 3720

(a) Operated by Battelle for the U.S. Department of Energy under contract DE-ACO6-76RLO 1830.

(b) The FEMPs have been updated over the years and are currently available for the 325,331 , 3720, and Balance-of-Plant Facilities (Pacific Northwest National Laboratory 199a,b,c,d). 
Building to the 331 Building upgraded the 331 Building to major stack status. The move was accomplished in 1998 and 1999 with disposal of some materials resulting in both the 3720 and the 331 Buildings Potential-To-Emit ${ }^{1}$ (PTE) dropping to less than $0.1 \mathrm{mrem}$. The major stack for 3720 may be downgraded to minor, but continued work in the 331 Building may result in increased throughput. Thus, the 331 Building main stack is maintained as a major stack and continuously sampled. Results of the 1992-1993 assessment were documented by Sula and Jette (1994), and results of the 1995 assessment were documented in Ballinger, Jette, and Sula (1995). This report documents the most recent methods and assessments for 1999.

\footnotetext{
${ }^{1}$ Potential-to-Emit is defined as the rate of release of radionuclides from an emission unit based on the actual or potential discharge of the effluent stream that would result if all abatement control equipment did not exist, but operations are otherwise normal.
} 


\subsection{Assessment Methodology}

Requirements for facility air emission sampling are promulgated by

- the U.S. Environmental Protection Agency (EPA) in 40 CFR 61, "National Emissions Standards for Hazardous Air Pollutants," Subpart H, "National Emission Standards for Emissions of Radionuclides Other than Radon from Department of Energy Facilities"

- the Washington State Department of Health in Washington Administrative Code 246-247, "Regulation of Radioactive Air Emissions"

- the DOE in regulatory guide DOE/EH-0173T, "Environmental Regulatory Guide for Radiological Monitoring and Environmental Surveillance."

These regulations require that all emission units with the potential to emit radioactivity be evaluated, and that the emission units be continuously sampled if there is the potential for unmitigated releases to cause a dose of $0.1 \mathrm{mrem} / \mathrm{yr}$ to a maximum offsite receptor.

\subsection{Projections of Annual Emission Quantities}

Several methods for projecting potential unmitigated annual emission quantities are prescribed in the 1994 issuance of WAC 246-247:

- apply an annual release fraction to the radionuclide inventory in the facility

- multiply actual measured annual emissions by control system decontamination factors

- add actual measured annual emission quantities to actual measured quantities retained by control systems

- measure the annual discharge upstream from all control devices.

The inventory-based assessment method ${ }^{(a)}$ has been used by Pacific Northwest since the initial facility assessment in 1991. Whereas the inventory method yields an assessment based on the current building status (or even the future status if projected future inventory quantities are used in the assessment), the other prescribed methods yield an assessment based on past facility

(a) This method is described in WAC 246-247 as follows: Multiply the annual possession quantity of each radionuclide by the release fraction for that radionuclide, depending on its physical state. Use the following release fractions: (i) 1 for gases, (ii) $10^{-3}$ for liquids or particulate solids, and (iii) $10^{-6}$ for solids. Determine the physical state for each radionuclide by considering its chemical form and the highest temperature to which it is subjected. Use a release fraction of 1 if the radionuclide is subjected to temperatures at or above its boiling point; use a release fraction of $10^{-3}$ if the radionuclide is subjected to temperatures at or above its melting point but below its boiling point. If the chemical form is not known, use a release fraction of 1 for any radionuclide that is heated to a temperature of one hundred degrees Celsius or more, boils at a temperature of one hundred degrees Celsius or less, or is intentionally dispersed into the environment. 
measurements. Thus, the inventory method may be more appropriate for use at research and development facilities where types and quantities of radionuclides may change from year to year and where historical sampling data may not be a reliable predictor of future emissions.

At Pacific Northwest, radioactive source and material information is maintained using three separate inventory systems:

Nuclear Materials Inventory. This inventory includes the majority of tritium, uranium, and transuranics in Pacific Northwest facilities. The inventory includes material in process as well as residual contamination from historical operations in the facility. The Nuclear Materials Inventory is categorized as "Type 3 inventory."

Composite Radioactive Materials Inventory. This inventory includes DOE-owned sealed radioactive sources above specific de minimus values (based on the December 1998 revision to 10 CFR 835) and all radioactive material that is possessed by Pacific Northwest under a State of Washington Radioactive Materials License. Most of the radionuclides in this inventory are in "sealed source" form. The Composite Radioactive Materials Inventory is categorized as "Type 2 inventory."

Facilities Management Radioactive Materials Inventory. This database was developed specifically to account for all radioactive material not included in either the Type 2 or Type 3 inventories. This category consists primarily of fission product radionuclides, including radionuclides in process as well as residual contamination (e.g., in hot cells) from historical operations in the facility. The Facilities Management Radioactive Material Inventory is categorized as "Type 1 inventory."

The Pacific Northwest Effluent Management Group annually requests Type 1 inventory information from the custodians of radioactive inventory. This request is made through a central point of contact for each of Pacific Northwest's Divisions who then request inventory information from custodians in their organization. Effluent Management also requests Type 2 and Type 3 inventories from the Composite Radioactive Materials Inventory and Nuclear Materials Inventory databases, respectively.

Although the type of information contained in each of the systems varies, after compiling the information and reformatting it, radioactive materials are identified by type, physical form, and quantity. Quantities are expressed either in terms of activity $(\mathrm{Ci})$ or mass (grams). Radioactive material types may be expressed as specific radionuclides or as standard mixtures of radionuclides. For example, mixed radionuclides may be expressed as natural uranium, depleted uranium, Hanford $6 \%\left({ }^{240} \mathrm{Pu}\right)$ plutonium, etc., as shown in Table 1. Appendix A provides the basis for these values. In addition to the mixtures shown in the table, mixtures of fission products are assumed to consist of ${ }^{90} \mathrm{Sr}$. Individual radionuclides or mixtures of radionuclides in small quantities may also be reported as alpha or beta activity for simplification. These materials are assumed to be ${ }^{241} \mathrm{Am}$ (alpha) or ${ }^{90} \mathrm{Sr}$ (beta) for dose assessment purposes. 
Table 1. Common Radionuclide Mixtures

\begin{tabular}{|c|c|c|}
\hline Material & Assumed Radionuclide & $\begin{array}{c}\text { Specific Activity } \\
\text { (Ci/g) }\end{array}$ \\
\hline Depleted Uranium & ${ }^{238} \mathrm{U}$ & $3.64 \mathrm{E}-07$ \\
\hline Natural Uranium & ${ }^{238} \mathrm{U}$ & $6.87 \mathrm{E}-07$ \\
\hline U-enriched $\left(<20 \%{ }^{235} \mathrm{U}\right)$ & ${ }^{235} \mathrm{U}$ & $9.36 \mathrm{E}-06$ \\
\hline U-enriched $\left(<90 \%{ }^{235} \mathrm{U}\right)$ & ${ }^{235} \mathrm{U}$ & $6.21 \mathrm{E}-05$ \\
\hline $\mathrm{Pu}\left(6 \%{ }^{240} \mathrm{Pu}\right)$ & ${ }^{239} \mathrm{Pu}$ & $8.00 \mathrm{E}-02$ \\
\hline $\mathrm{Pu}\left(12 \%{ }^{240} \mathrm{Pu}\right)$ & ${ }^{239} \mathrm{Pu}$ & $9.60 \mathrm{E}-02$ \\
\hline $\mathrm{Pu}\left(24 \%{ }^{240} \mathrm{Pu}\right)$ & ${ }^{239} \mathrm{Pu}$ & $3.48 \mathrm{E}-01$ \\
\hline
\end{tabular}

Radionuclides meeting any of the following criteria are excluded from the assessments:

- radionuclides present in commercially available building/construction materials

- radionuclides that can be purchased or possessed without a special radioactive materials license

- radionuclides $<100 \mathrm{pCi} / \mathrm{g}$ alpha activity and $<400 \mathrm{pCi} / \mathrm{g}$ beta activity.

Inventory data is entered into an ACCESS database that was developed specifically for these calculations. Appendix B provides a summary of the database features. The data are reviewed and revised as needed to eliminate duplicates, provide consistency in nuclide and unit identifications, and obtain additional information as required by the calculations. The review process is documented and filed with the assessment records.

Potential release fractions for radionuclides are based on the physical form of the radionuclide as shown in Table 2. Radionuclides present as sealed sources or in sealed, unvented Department of Transportation (DOT) shipping containers are assumed to be unavailable for release under normal circumstances. 
Table 2. Physical Forms and Potential Annual Release Fractions for Radionuclides

\begin{tabular}{|c|c|c|c|}
\hline Form & Code & Description & $\begin{array}{c}\text { Potential } \\
\text { Release } \\
\text { Fraction }\end{array}$ \\
\hline Gas & G & $\begin{array}{l}\text { Nuclide will exceed its boiling point when } \\
\text { uncontained, except that nuclides in gaseous } \\
\text { form in commercial gas cylinders that are not } \\
\text { opened may be listed as L. }\end{array}$ & 1 \\
\hline Liquid/Powder & $\mathrm{L} / \mathrm{P}$ & $\begin{array}{l}\text { Nuclide will exceed its melting point or be } \\
\text { present in particulate form (Aerodynamic Mean } \\
\text { Diameter [AMD] }<100 \text { microns) when } \\
\text { uncontained, except liquid and powders in } \\
\text { unopened containers may be listed as S. }\end{array}$ & $10^{-3}$ \\
\hline Solid & $S$ & $\begin{array}{l}\text { Nuclides not meeting conditions for the more } \\
\text { dispersible classes. }\end{array}$ & $10^{-6}$ \\
\hline Contained & $\mathrm{C}$ & $\begin{array}{l}\text { Sealed sources or material in sealed, DOT } \\
\text { containers, except those meeting exempt criteria. }\end{array}$ & 0 \\
\hline Exempt & $\mathrm{E}$ & $\begin{array}{l}\text { Sealed sources engineered to pass the special } \\
\text { form testing specified by the DOT in } \\
40 \mathrm{CFR} 173.469 \text { or ANSI }{ }^{(a)} \mathrm{N} 43.6 \text {, or sealed in } \\
\text { Type B DOT shipping containers. }\end{array}$ & 0 \\
\hline
\end{tabular}

(a) American National Standards Institute.

\subsection{Maximum Receptor Unit Dose Calculation}

For the unit dose calculations, the maximum offsite receptor is defined as an individual whose residence location, work location, and lifestyle maximize the dose from airborne pathways. All potential environmental transport pathways associated with an airborne radionuclide release were included (i.e., air inhalation, air submersion, exposure to deposited radionuclides, uptake of vegetation grown in contaminated soil). In addition, site-specific atmospheric dispersion and environmental transport and uptake parameters were used (see Appendix C).

Unit dose factors for the maximum offsite receptor were calculated for specific radionuclides using the EPA compliance code CAP88-PC (Parks 1992). For radionuclides that were not represented in CAP88-PC, GENII (Napier et al. 1988) was used to calculate unit doses. Radionuclides that were not represented in either CAP88-PC or GENII were assigned default values equal to that of ${ }^{241} \mathrm{Am}$ for alpha emitters or ${ }^{90} \mathrm{Sr}$ for non-alpha emitters. The decay of the daughter products was also considered for short half-life radionuclides in assigning default 
values. The unit dose factor calculations were performed for a single source point in the 300 Area of the Hanford Site (Table 3) and a single source point in the 200 East Area of the Site (WHC 1991).

For Building 6652 (located in the Fitzner/Eberhardt Arid Land Ecology [ALE] Reserve), dose assessments were performed by applying a location correction factor to the 300 Area unit dose factor to correct for varying source-receptor distances and directions. The location correction factor was calculated by dividing the atmospheric dispersion values $(\mathrm{Chi} / \mathrm{Q})$ for 6652 by the atmospheric dispersion values for the 300 Area. The code CAP88-PC was used to calculate these dispersion values. Similar calculations were performed to obtain location correction factors for other Pacific Northwest-operated DOE facilities outside of the 300 Area.

\subsection{Potential Emission Dose Assessment}

Doses from projected radionuclide emissions were calculated by multiplying the quantity of each radionuclide present in the facility by its associated potential release fraction, the 300 Area unit dose factor (see Appendix C), and the location correction factor. Doses from individual radionuclides were summed to derive the total potential annual emission dose for each facility.

The assessments are independently reviewed, then packets are prepared for each building containing the raw inventory information, any communications clarifying or correcting the inventory information, summarized inventory information, and a cover sheet showing the resulting dose and approval signatures. The packets are approved by the preparer, the reviewer, the divisional points of contact for the inventory custodians, and the building managers. Once approved, assessment packets are maintained as records by the Effluent Management Group. A summary of the results of the assessment for 1999 and the primary contributing radionuclides for facilities that require continuous monitoring is provided in Table 4 . The table also identifies the radionuclides that contribute $10 \%$ or more of the potential dose for facilities that are required to be continuously sampled. 
Table 3. Unit Dose Factors for 300 Area Source Location

\begin{tabular}{|c|c|c|c|c|c|}
\hline Nuclide & Dose $(\mathrm{mrem} / \mathrm{Ci})$ & Nuclide & Dose $(\mathrm{mrem} / \mathrm{Ci})$ & Nuclide & Dose $(\mathrm{mrem} / \mathrm{Ci})$ \\
\hline $\mathrm{Ac}-225$ & $3.10 \mathrm{E}-00$ & $\mathrm{Co}-57$ & $6.10 \mathrm{E}-02$ & $\mathrm{Kr}-89$ & $7.60 \mathrm{E}-05$ \\
\hline Ac-227 & $3.30 \mathrm{E}+02$ & Co-58 & $1.20 \mathrm{E}-01$ & $\mathrm{Kr}-90$ & $3.80 \mathrm{E}-08$ \\
\hline Ac-228 & $3.80 \mathrm{E}-02$ & Co-60 & $5.00 \mathrm{E}-00$ & La-140 & $9.30 \mathrm{E}-03$ \\
\hline Ag-109m & $5.50 \mathrm{E}-10$ & $\mathrm{Cr}-51$ & $2.30 \mathrm{E}-03$ & La-141 & $1.80 \mathrm{E}-05$ \\
\hline $\mathrm{Ag}-110$ & $6.40 \mathrm{E}-11$ & Cs-134 & $2.10 \mathrm{E}-00$ & La-142 & $9.20 \mathrm{E}-04$ \\
\hline $\mathrm{Ag}-110 \mathrm{~m}$ & $9.60 \mathrm{E}-01$ & Cs-134m & $2.90 \mathrm{E}-05$ & $\mathrm{Mn}-54$ & $3.40 \mathrm{E}-01$ \\
\hline $\mathrm{Ag}-111$ & $1.90 \mathrm{E}-02$ & Cs-135 & $1.20 \mathrm{E}-01$ & $M n-56$ & $8.70 \mathrm{E}-04$ \\
\hline $\mathrm{Am}-241$ & $2.90 \mathrm{E}+02$ & Cs-136 & $8.80 \mathrm{E}-02$ & Mo-93 & $5.60 \mathrm{E}-02$ \\
\hline Am-242 & $2.70 \mathrm{E}-02$ & Cs-137 & $4.90 \mathrm{E}-00$ & Mo-99 & $5.20 \mathrm{E}-03$ \\
\hline Am-242m & $2.80 \mathrm{E}+02$ & Cs-138 & $5.90 \mathrm{E}-04$ & $\mathrm{~N}-13$ & $1.30 \mathrm{E}-04$ \\
\hline Am-243 & $2.90 \mathrm{E}+02$ & Cs-139 & $4.00 \mathrm{E}-05$ & $\mathrm{Na}-22$ & $2.40 \mathrm{E}-00$ \\
\hline Ar-41 & $3.30 \mathrm{E}-04$ & Cu-64 & $3.40 \mathrm{E}-04$ & $\mathrm{Na}-24$ & $4.50 \mathrm{E}-03$ \\
\hline As-76 & $3.00 \mathrm{E}-03$ & Eu-152 & $4.90 \mathrm{E}-00$ & $\mathrm{Nb}-93 \mathrm{~m}$ & $2.80 \mathrm{E}-02$ \\
\hline $\mathrm{Ba}-133$ & $1.50 \mathrm{E}-00$ & Eu-152m & $2.30 \mathrm{E}-04$ & $\mathrm{Nb}-94$ & $1.60 \mathrm{E}+01$ \\
\hline $\mathrm{Ba}-133 \mathrm{~m}$ & $8.60 \mathrm{E}-04$ & Eu-154 & $4.00 \mathrm{E}-00$ & $\overline{\mathrm{Nb}-95}$ & $6.40 \mathrm{E}-02$ \\
\hline Ba-137m & $1.50 \mathrm{E}-05$ & $\overline{E u-155}$ & $1.70 \mathrm{E}-01$ & $\mathrm{Nb}-95 \mathrm{~m}$ & $4.60 \mathrm{E}-03$ \\
\hline Ba-139 & $8.80 \mathrm{E}-05$ & Eu-156 & $7.40 \mathrm{E}-02$ & Nb-97 & $2.50 \mathrm{E}-04$ \\
\hline Ba-140 & $7.90 \mathrm{E}-02$ & $\mathrm{~F}-18$ & $3.60 \mathrm{E}-04$ & Nb-97m & $9.40 \mathrm{E}-07$ \\
\hline Ba-141 & $1.60 \mathrm{E}-04$ & $\mathrm{Fe}-55$ & $6.90 \mathrm{E}-03$ & Nd-147 & $2.20 \mathrm{E}-02$ \\
\hline Ba-142 & $1.20 \mathrm{E}-04$ & Fe-59 & $1.20 \mathrm{E}-01$ & $\mathrm{Ni}-59$ & $8.10 \mathrm{E}-03$ \\
\hline $\mathrm{Be}-7$ & $4.50 \mathrm{E}-03$ & Fr-221 & $4.90 \mathrm{E}-04$ & Ni-63 & $1.00 \mathrm{E}-02$ \\
\hline Bi-210 & $9.90 \mathrm{E}-02$ & Fr-223 & $8.30 \mathrm{E}-04$ & $\mathrm{Ni}-65$ & $3.50 \mathrm{E}-04$ \\
\hline $\mathrm{Bi}-211$ & $3.20 \mathrm{E}-05$ & Ga-67 & $1.70 \mathrm{E}-03$ & $\mathrm{~Np}-237$ & $2.60 \mathrm{E}+02$ \\
\hline $\mathrm{Bi}-212$ & $1.30 \mathrm{E}-02$ & $\mathrm{H}-3$ & $4.10 \mathrm{E}-04$ & Np-238 & $1.70 \mathrm{E}-02$ \\
\hline$B i-213$ & $4.90 \mathrm{E}-04$ & $\mathrm{Hf}-181$ & $7.00 \mathrm{E}-02$ & Np-239 & $3.40 \mathrm{E}-03$ \\
\hline Bi-214 & $4.10 \mathrm{E}-04$ & $\mathrm{Hg}-203$ & $4.40 \mathrm{E}-02$ & Np-240 & $3.70 \mathrm{E}-04$ \\
\hline $\mathrm{Br}-82$ & $6.30 \mathrm{E}-03$ & Ho-166 & $2.10 \mathrm{E}-03$ & \begin{tabular}{|l|}
$\mathrm{Np}-240 \mathrm{~m}$ \\
\end{tabular} & $3.70 \mathrm{E}-05$ \\
\hline $\mathrm{Br}-83$ & $2.70 \mathrm{E}-06$ & Ho-166m & $1.60 \mathrm{E}+01$ & $0-15$ & $1.50 \mathrm{E}-05$ \\
\hline $\mathrm{Br}-84$ & $4.30 \mathrm{E}-04$ & I-122 & $2.50 \mathrm{E}-05$ & P-32 & $5.70 \mathrm{E}-02$ \\
\hline $\mathrm{Br}-85$ & $2.10 \mathrm{E}-06$ & I-123 & $1.20 \mathrm{E}-03$ & $\mathrm{~Pa}-231$ & $2.70 \mathrm{E}+02$ \\
\hline$C-11$ & $1.90 \mathrm{E}-04$ & $1-125$ & $2.80 \mathrm{E}-00$ & \begin{tabular}{|l|}
$\mathrm{Pa}-233$ \\
\end{tabular} & $3.50 \mathrm{E}-02$ \\
\hline $\mathrm{C}-14$ & $5.00 \mathrm{E}-02$ & $1-129$ & $4.80 \mathrm{E}+01$ & \begin{tabular}{|l|}
$\mathrm{Pa}-234$ \\
\end{tabular} & $1.70 \mathrm{E}-03$ \\
\hline $\mathrm{Ca}-41$ & $2.00 \mathrm{E}-04$ & $1-130$ & $1.30 \mathrm{E}-02$ & \begin{tabular}{|l|}
$\mathrm{Pa}-234 \mathrm{~m}$ \\
\end{tabular} & $4.50 \mathrm{E}-08$ \\
\hline Cd-109 & \begin{tabular}{l|l}
$1.20 \mathrm{E}-01$ & $*$
\end{tabular} & $1-131$ & $1.40 \mathrm{E}-00$ & $\mathrm{~Pb}-209$ & $4.30 \mathrm{E}-05$ \\
\hline Cd-115 & $5.20 \mathrm{E}-03$ & $1-132$ & $2.50 \mathrm{E}-03$ & $\mathrm{~Pb}-210$ & $6.30 \mathrm{E}+01$ \\
\hline $\mathrm{Cd}-115 \mathrm{~m}$ & $1.40 \mathrm{E}-01$ & $1-133$ & $1.00 \mathrm{E}-02$ & $\mathrm{~Pb}-211$ & $3.40 \mathrm{E}-03$ \\
\hline $\mathrm{Ce}-141$ & $2.70 \mathrm{E}-02$ & $\mathrm{I}-134$ & $1.10 \mathrm{E}-03$ & $\mathrm{~Pb}-212$ & $8.10 \mathrm{E}-02$ \\
\hline $\mathrm{Ce}-143$ & $2.80 \mathrm{E}-03$ & $\mathrm{I}-135$ & $4.60 \mathrm{E}-03$ & $\mathrm{~Pb}-214$ & $3.60 \mathrm{E}-04$ \\
\hline Ce-144 & $3.70 \mathrm{E}-01$ & $\operatorname{In-113m}$ & $1.00 \mathrm{E}-04$ & \begin{tabular}{|l|}
$\mathrm{Pd}-107$ \\
\end{tabular} & $8.50 \mathrm{E}-03$ \\
\hline \begin{tabular}{|l|}
$\mathrm{Cf}-252$ \\
\end{tabular} & $7.90 \mathrm{E}+01$ & $\ln -115$ & $2.00 \mathrm{E}-00$ & $\begin{array}{l}\mathrm{Pd}-109 \\
\end{array}$ & $7.60 \mathrm{E}-04$ \\
\hline $\mathrm{Cl}-36$ & \begin{tabular}{|l|l}
$2.20 \mathrm{E}-01$ & $*$
\end{tabular} & In-115m & $1.50 \mathrm{E}-04$ & Pm-147 & $2.80 \mathrm{E}-02$ \\
\hline $\mathrm{Cm}-242$ & $9.10 \mathrm{E}-00$ & Ir-192 & $1.40 \mathrm{E}-01$ & Pm-148 & $3.00 \mathrm{E}-02$ \\
\hline $\mathrm{Cm}-243$ & $1.90 \mathrm{E}+02$ & $\mathrm{~K}-40$ & $3.30 \mathrm{E}-00$ & Pm-148m & $2.00 \mathrm{E}-01$ \\
\hline $\mathrm{Cm}-244$ & $1.50 \mathrm{E}+02$ & $\mathrm{Kr}-83 \mathrm{~m}$ & $6.00 \mathrm{E}-08$ & \begin{tabular}{|l|}
$\mathrm{Pm}-149$ \\
\end{tabular} & $3.20 \mathrm{E}-03$ \\
\hline $\mathrm{Cm}-245$ & $3.00 \mathrm{E}+02$ & $\mathrm{Kr}-85$ & $9.20 \mathrm{E}-07$ & $\mathrm{Pm}-151$ & $6.00 \mathrm{E}-04$ \\
\hline $\mathrm{Cm}-246$ & $2.90 \mathrm{E}+02$ & $\mathrm{Kr}-85 \mathrm{~m}$ & $4.30 \mathrm{E}-05$ & Po-210 & $2.10 \mathrm{E}+01$ \\
\hline $\mathrm{Cm}-247$ & $2.70 \mathrm{E}+02$ & $\mathrm{Kr}-87$ & $2.10 \mathrm{E}-04$ & Po-218 & $3.10 \mathrm{E}-06$ \\
\hline $\mathrm{Cm}-248$ & $1.10 \mathrm{E}+03$ & $\mathrm{Kr}-88$ & $5.90 \mathrm{E}-04$ & Pr-143 & $2.50 \mathrm{E}-02$ \\
\hline
\end{tabular}




\begin{tabular}{|c|c|c|c|c|c|}
\hline Nuclide & Dose $(\mathrm{mrem} / \mathrm{Ci})$ & Nuclide & Dose $(\mathrm{mrem} / \mathrm{Ci})$ & Nuclide & Dose $(\mathrm{mrem} / \mathrm{Ci})$ \\
\hline Pr-144 & $2.80 \mathrm{E}-05$ & Sn-126 & $9.00 \mathrm{E}-01$ & Xe-135 & $6.70 \mathrm{E}-05$ \\
\hline Pr-144m & $6.30 \mathrm{E}-06$ & Sr-89 & $7.60 \mathrm{E}-02$ & $\mathrm{Xe}-135 \mathrm{~m}$ & $6.80 \mathrm{E}-05$ \\
\hline Pu-236 & $4.40 \mathrm{E}+01$ & Sr-90 & $4.90 \mathrm{E}-00$ & $\mathrm{Xe}-137$ & $9.80 \mathrm{E}-06$ \\
\hline $\mathrm{Pu}-238$ & $1.80 \mathrm{E}+02$ & Sr-91 & $1.00 \mathrm{E}-03$ & $\mathrm{Xe}-138$ & $1.90 \mathrm{E}-04$ \\
\hline Pu-239 & $2.00 \mathrm{E}+02$ & Sr-92 & $7.80 \mathrm{E}-04$ & $\mathrm{Y}-90$ & $1.10 \mathrm{E}-02$ \\
\hline $\mathrm{Pu}-240$ & $2.00 \mathrm{E}+02$ & $\mathrm{~Tb}-160$ & $1.60 \mathrm{E}-01$ & $\mathrm{Y}-90 \mathrm{~m}$ & $2.60 \mathrm{E}-04$ \\
\hline Pu-241 & $3.20 \mathrm{E}+02$ & Tc-101 & $5.20 \mathrm{E}-05$ & Y-91 & $9.80 \mathrm{E}-02$ \\
\hline $\mathrm{Pu}-242$ & $1.80 \mathrm{E}+02$ & Tc-97 & $1.30 \mathrm{E}-01$ & $\bar{Y}-91 \mathrm{~m}$ & $1.50 \mathrm{E}-04$ \\
\hline Pu-243 & $1.10 \mathrm{E}-04$ & Tc-99 & $6.20 \mathrm{E}-01$ & Y-92 & $6.30 \mathrm{E}-04$ \\
\hline $\mathrm{Pu}-244$ & $1.80 \mathrm{E}+02$ & Te-125m & $3.50 \mathrm{E}-02$ & Y-93 & $1.30 \mathrm{E}-03$ \\
\hline Ra-223 & $6.60 \mathrm{E}-00$ & Te-127 & $1.90 \mathrm{E}-04$ & $\mathrm{Zn}-65$ & $4.30 \mathrm{E}-01$ \\
\hline Ra-224 & $2.00 \mathrm{E}-00$ & Te-127m & $8.50 \mathrm{E}-02$ & $\mathrm{Zn}-69$ & $2.60 \mathrm{E}-05$ \\
\hline Ra-225 & $4.70 \mathrm{E}-00$ & Te-129 & $6.80 \mathrm{E}-05$ & $2 n-69 m$ & $8.80 \mathrm{E}-04$ \\
\hline Ra-226 & $1.70 \mathrm{E}+01$ & $\mathrm{Te}-129 \mathrm{~m}$ & $8.60 \mathrm{E}-02$ & Zr-93 & $3.20 \mathrm{E}-02$ \\
\hline Ra-228 & $7.90 \mathrm{E}-00$ & Te-131 & $1.20 \mathrm{E}-04$ & Zr-95 & $1.30 \mathrm{E}-01$ \\
\hline $\mathrm{Rb}-86$ & $7.20 \mathrm{E}-02$ & Te-131m & $5.20 \mathrm{E}-03$ & & \\
\hline $\mathrm{Rb}-87$ & $2.60 \mathrm{E}-01$ & Te-132 & $1.30 \mathrm{E}-02$ & & \\
\hline$\overline{\mathrm{Rb}-88}$ & $1.50 \mathrm{E}-04$ & Te-133 & $1.40 \mathrm{E}-04$ & & \\
\hline $\mathrm{Rb}-89$ & $3.70 \mathrm{E}-04$ & Te-133m & $6.00 \mathrm{E}-04$ & & \\
\hline Rb-90 & $6.60 \mathrm{E}-05$ & Te-134 & $2.10 \mathrm{E}-04$ & & \\
\hline $\mathrm{Rb}-90 \mathrm{~m}$ & $2.10 \mathrm{E}-04$ & Th-227 & $5.50 \mathrm{E}-00$ & & \\
\hline $\operatorname{Re}-187$ & $6.40 \mathrm{E}-04$ & Th-228 & $1.20 \mathrm{E}+02$ & & \\
\hline $\mathrm{Rh}-103 \mathrm{~m}$ & $4.90 \mathrm{E}-00$ & Th-229 & $3.20 \mathrm{E}+02$ & & \\
\hline Rh-105 & $9.40 \mathrm{E}-04$ & Th-230 & $1.20 \mathrm{E}+02$ & & \\
\hline $\mathrm{Rh}-105 \mathrm{~m}$ & $7.80 \mathrm{E}-09$ & Th-231 & $5.40 \mathrm{E}-04$ & & \\
\hline Rh-106 & $2.80 \mathrm{E}-09$ & Th-232 & $1.90 \mathrm{E}+02$ & & \\
\hline $\mathrm{Rn}-219$ & $1.40 \mathrm{E}-34$ & Th-234 & $9.90 \mathrm{E}-02$ & & \\
\hline $\mathrm{Rn}-220$ & $5.50 \mathrm{E}-07$ & Tl-207 & $1.10 \mathrm{E}-06$ & & \\
\hline $\mathrm{Rn}-222$ & $1.20 \mathrm{E}-03$ & Tl-208 & $1.40 \mathrm{E}-04$ & & \\
\hline Ru-103 & $5.00 \mathrm{E}-02$ & T1-209 & $3.90 \mathrm{E}-05$ & & \\
\hline Ru-105 & $6.60 \mathrm{E}-04$ & $\overline{\mathrm{U}-232}$ & $2.40 \mathrm{E}+02$ & & \\
\hline $\mathrm{Ru}-106$ & $4.70 \mathrm{E}-01$ & U-233 & $7.10 \mathrm{E}+01$ & & \\
\hline Ru-97 & $1.60 \mathrm{E}-03$ & U-234 & $7.00 \mathrm{E}+01$ & & \\
\hline $\mathrm{S}-35$ & $6.20 \mathrm{E}-03$ & U-235 & $6.70 \mathrm{E}+01$ & & \\
\hline Sb-124 & $2.20 \mathrm{E}-01$ & U-236 & $6.60 \mathrm{E}+01$ & & \\
\hline Sb-125 & \begin{tabular}{|l|}
$5.40 \mathrm{E}-01$ \\
\end{tabular} & U-237 & $1.10 \mathrm{E}-02$ & & \\
\hline $\mathrm{Sb}-126$ & $9.10 \mathrm{E}-02$ & U-238 & $6.20 \mathrm{E}+01$ & & \\
\hline Sb-126m & $3.00 \mathrm{E}-04$ & $\overline{U-240}$ & \begin{tabular}{|c|}
$1.10 \mathrm{E}-03$ \\
\end{tabular} & & \\
\hline $\mathrm{Sb}-127$ & $1.40 \mathrm{E}-02$ & W-181 & $9.50 \mathrm{E}-03$ & & \\
\hline Sc-46 & $2.70 \mathrm{E}-01$ & W-185 & $1.50 \mathrm{E}-02$ & & \\
\hline Se-75 & $9.10 \mathrm{E}-01$ & W-187 & $1.10 \mathrm{E}-03$ & & \\
\hline Se-79 & $1.70 \mathrm{E}-00$ & $\mathrm{Xe}-122$ & $1.80 \mathrm{E}-05$ & & \\
\hline Sm-147 & $3.60 \mathrm{E}+01$ & $\mathrm{Xe}-123$ & $1.60 \mathrm{E}-04$ & & \\
\hline $\mathrm{Sm}-151$ & $1.80 \mathrm{E}-02$ & $\mathrm{Xe}-125$ & $6.80 \mathrm{E}-05$ & & \\
\hline Sm-153 & $2.10 \mathrm{E}-03$ & $\mathrm{Xe}-127$ & $7.20 \mathrm{E}-05$ & & \\
\hline Sn-113 & $4.00 \mathrm{E}-02$ & $\mathrm{Xe}-131 \mathrm{~m}$ & $2.60 \mathrm{E}-06$ & & \\
\hline Sn-123 & $1.00 \mathrm{E}-03$ & $\mathrm{Xe}-133$ & $9.60 \mathrm{E}-06$ & & \\
\hline$\overline{S n-125}$ & $5.70 \mathrm{E}-02$ & $\mathrm{Xe}-133 \mathrm{~m}$ & $8.40 \mathrm{E}-06$ & & \\
\hline
\end{tabular}

* Calculated using the GENII Code. 
Table 4. Emission System Potential Dose Assessment Summary

\begin{tabular}{|c|c|c|c|c|c|}
\hline $\begin{array}{l}\text { Emission } \\
\text { System }\end{array}$ & \begin{tabular}{|l} 
Emission \\
Type $^{1}$
\end{tabular} & System Description & \begin{tabular}{|l} 
Emission \\
Measurement \\
Required
\end{tabular} & $\begin{array}{l}\text { Nuclides } \\
\text { Contributing }> \\
10 \% \text { of Potential } \\
\text { Dose }\end{array}$ & Comment \\
\hline \multicolumn{6}{|c|}{ Systems Located in the Southeast Region (300 Area) of the Hanford Site } \\
\hline $300-\mathrm{N}$ & Fugitive & Caisson & None & None & $\begin{array}{l}\text { Sealed } \\
\text { Sources Only }\end{array}$ \\
\hline $305-\mathrm{B}$ & $\begin{array}{l}\text { Fugitive } \\
+ \text { Point }\end{array}$ & $\begin{array}{l}\text { Hazardous Waste Storage } \\
\text { Facility }\end{array}$ & Confirmatory & $\mathrm{Pu}-238$ & \\
\hline 306-W & Point & $\begin{array}{l}\text { Materials Development } \\
\text { Building }\end{array}$ & Confirmatory & $\begin{array}{l}\mathrm{U}(20 \%) \\
\mathrm{U}(\text { depleted })\end{array}$ & \\
\hline 314 & $\begin{array}{l}\text { Fugitive } \\
+ \text { Point }\end{array}$ & $\begin{array}{l}\text { Engineering Development } \\
\text { Laboratory }\end{array}$ & None & U(natural) & $\begin{array}{l}\text { Bldg vacated } \\
\text { (Inventory } \\
\text { based on } \\
\text { holdup) }\end{array}$ \\
\hline 318 & Point & $\begin{array}{l}\text { Radiological Calibrations } \\
\text { Laboratory }\end{array}$ & None & Eu-154, U(natural) & $\begin{array}{l}\text { Primarily } \\
\text { sealed sources }\end{array}$ \\
\hline 320 & Point & $\begin{array}{l}\text { Analytical and Nuclear } \\
\text { Research Laboratory }\end{array}$ & Confirmatory & U-233 & \\
\hline 323 & Point & $\begin{array}{l}\text { Mechanical Properties } \\
\text { Laboratory }\end{array}$ & Confirmatory & Co-60, V-49 & \\
\hline 325 & Point & $\begin{array}{l}\text { Radiochemical Processing } \\
\text { Laboratory }\end{array}$ & Continuous & $\mathrm{H}-3, \mathrm{Pu}-238$ & \\
\hline 326 & Point & $\begin{array}{l}\text { Materials Sciences } \\
\text { Laboratory }\end{array}$ & Confirmatory & Gd-149, U(natural) & \\
\hline 329 & Point & $\begin{array}{l}\text { Chemical Sciences } \\
\text { Laboratory }\end{array}$ & Confirmatory & $\mathrm{Am}-241$ & \\
\hline 331 & Point & Life Sciences Laboratory I & Continuous & $\mathrm{Pu}-339, \mathrm{Pu}-241$ & \\
\hline $331 \mathrm{G}$ & Point & Interim Tissue Repository & Confirmatory & $\mathrm{C}-14$ & \\
\hline $331 \mathrm{H}$ & Point & $\begin{array}{l}\text { Aerosol Wind Tunnel } \\
\text { Research Facility }\end{array}$ & None & None & $\begin{array}{l}\text { Sealed } \\
\text { Sources Only }\end{array}$ \\
\hline $3718 \mathrm{~A}$ & Fugitive & Lab Equipment Central Pool & None & U(natural) & \\
\hline 3720 & Point & $\begin{array}{l}\text { Environmental Sciences } \\
\text { Laboratory }\end{array}$ & Continuous & $\mathrm{Am}-243, \mathrm{Pu}-238$ & $\begin{array}{l}1999 \text { PTE < } \\
0.1 \text { mrem; } \\
\text { facility in } \\
\text { transition }\end{array}$ \\
\hline 3730 & Point & Gamma Irradiation Facility & Confirmatory & Co- $60, \mathrm{~V}-49$ & \\
\hline 3745 & Point & $\begin{array}{l}\text { Radiological Calibrations } \\
\text { and Standards Building }\end{array}$ & Confirmatory & $\mathrm{U}$ (Hanford) & \\
\hline $\begin{array}{l}3020 \\
\text { EMSL }\end{array}$ & Point & $\begin{array}{l}\text { Environmental Molecular } \\
\text { Sciences Laboratory }\end{array}$ & None & None & $\begin{array}{l}\text { Sealed } \\
\text { Sources Only }\end{array}$ \\
\hline
\end{tabular}




\begin{tabular}{|c|c|c|c|c|c|}
\hline \multicolumn{6}{|c|}{ Systems Located in the Central Region (200 Areas) of the Hanford Site } \\
\hline $200 \mathrm{E}$ & Fugitive & $\begin{array}{l}\text { Environmental Monitoring } \\
\text { Shed }\end{array}$ & None & None & $\begin{array}{l}\text { Sealed } \\
\text { Sources Only }\end{array}$ \\
\hline FLTF & Fugitive & $\begin{array}{l}\text { Field Lysimeter Test } \\
\text { Facility }\end{array}$ & None & None & $\begin{array}{l}\text { Sealed } \\
\text { Sources Only }\end{array}$ \\
\hline$\overline{\mathrm{PSB}}$ & Fugitive & Prototype Surface Barrier & None & None & $\begin{array}{l}\text { Sealed } \\
\text { Sources Only }\end{array}$ \\
\hline $2718 \mathrm{E}$ & Fugitive & Critical Mass Fissile Storage & None & None & $\begin{array}{l}\text { Radioactive } \\
\text { Material in } \\
\text { DOT } \\
\text { containers }\end{array}$ \\
\hline$\overline{622 \mathrm{R}}$ & Fugitive & Meteorological Laboratory & None & None & $\begin{array}{l}\text { Sealed } \\
\text { Sources Only }\end{array}$ \\
\hline \multicolumn{6}{|c|}{ Systems Located on ALE } \\
\hline 6652 & Point & $\begin{array}{l}\text { Arid Land Ecology } \\
\text { Laboratory }\end{array}$ & None & None & $\begin{array}{l}\text { Sealed } \\
\text { Sources Only }\end{array}$ \\
\hline \multicolumn{6}{|c|}{ Systems Located in Richland } \\
\hline $747 \mathrm{~A}$ & Fugitive & Whole Body Counter & None & None & $\begin{array}{l}\text { Primarily } \\
\text { Sealed } \\
\text { Sources }\end{array}$ \\
\hline
\end{tabular}

1 "Fugitive emissions" are radioactive air emissions that do not and could not reasonably pass through a stack, vent, or other functionally equivalent structure, and that are not feasible to directly measure and quantify (WAC 246-247). 


\subsection{References}

10 CFR 835 1998. "Occupational Radiation Protection." U.S. Environmental Protection Agency, Washington, D.C.

40 CFR 61 1989. "National Emission Standards for Hazardous Air Pollutants." U.S. Environmental Protection Agency, Washington, D.C.

Ballinger, M. Y., S. J. Jette, and M. J. Sula. 1995. Assessment of Unabated Facility Emission Potentials for Evaluating Airborne Radionuclide Monitoring Requirements at Pacific Northwest National Laboratory - 1995. PNL-10855, Pacific Northwest Laboratory, Richland, Washington.

DOE. 1988. General Environmental Protection Program, DOE 5400.1, U.S. Department of Energy Order. U.S. Department of Energy, Washington, D.C.

DOE. 1991. Environmental Regulatory Guide for Radiological Effluent Monitoring and Environmental Surveillance, DOE/EH-0173T. U.S. Department of Energy, Washington, D.C.

EPA. 1990. National Emission Standards for Hazardous Air Pollutants. 40 CFR 61. Environmental Protection Agency, U.S. Code of Federal Regulations.

Napier, B. A., R. A. Peloquin, D. L. Strenge, and J. V. Ramsdell. 1988. GENII - The Hanford Environmental Radiation Dosimetry Software System. PNL-6584, Vols. 1-3. Pacific Northwest Laboratory, Richland, Washington.

Pacific Northwest National Laboratory. 1999a. Facility Effluent Monitoring Plan for the 325 Radiochemical Processing Laboratory. PNNL-12157. Pacific Northwest National Laboratory, Richland, Washington.

Pacific Northwest National Laboratory. 1999b. Facility Effluent Monitoring Plan for the 331 Complex. PNNL-12158. Pacific Northwest National Laboratory, Richland, Washington.

Pacific Northwest National Laboratory. 1999c. Facility Effluent Monitoring Plan for the 3720 Building. PNNL-12159. Pacific Northwest National Laboratory, Richland, Washington.

Pacific Northwest National Laboratory. 1999d. Facility Effluent Monitoring Plan for the Balance-of-Plant Facilities. PNNL-12160. Pacific Northwest National Laboratory, Richland, Washington.

Parks, B. S. 1992. User's Guide for CAP88-PC Version 1.0. 402-B-92-001, U.S. Environmental Protection Agency, Office of Radiation Programs, Las Vegas Facility, Las Vegas, Nevada.

Sula, M. J. and S. J. Jette. 1994. Pacific Northwest Laboratory Facilities Radionuclide 
Inventory Assessment CY 1992-1993. PNL-10061. Pacific Northwest Laboratory, Richland, Washington.

WAC. 1994. Radiation Protection - Air Emissions, WAC 246-247, Washington Administrative Code. Washington Department of Health.

Westinghouse Hanford Company. 1991. Unit Dose Calculation Methods and Summary of Facility Effluent Monitoring Plan Determinations. WHC-EP-0498. Westinghouse Hanford Company, Richland, Washington. 


\section{Appendix A}

\section{Common Radionuclide Mixtures}

\begin{tabular}{|c|c|c|c|c|}
\hline \multirow[t]{7}{*}{ Weapons Grade (6\% Pu-240) } & Isotope & wt fraction & $\begin{array}{c}\text { Isotope } \\
\mathrm{Ci} / \mathrm{g}\end{array}$ & $\begin{array}{c}\text { Mixture } \\
\mathrm{Ci} / \mathrm{g}\end{array}$ \\
\hline & Pu-238 & 0.0005 & 17.5 & 0.01 \\
\hline & Pu-239 & 0.93 & 0.062 & 0.06 \\
\hline & Pu-240 & 0.061 & 0.226 & 0.01 \\
\hline & $\overline{\mathrm{Pu}-242}$ & 0.0005 & 0.0039 & 0.00 \\
\hline & Am-241 & 0 & 3.24 & 0.00 \\
\hline & & & & $8.02 \mathrm{E}-02$ \\
\hline \multirow[t]{7}{*}{ Fuel Grade (12\% Pu-240) } & Isotope & wt fraction & $\begin{array}{c}\text { Isotope } \\
\mathrm{Ci} / \mathrm{g}\end{array}$ & $\begin{array}{c}\text { Mixture } \\
\mathrm{Cj} / \mathrm{g}\end{array}$ \\
\hline & $\overline{P u-238}$ & 0.001 & 17.1 & 0.02 \\
\hline & Pu-239 & 0.844 & 0.062 & 0.05 \\
\hline & Pu-240 & 0.124 & 0.226 & 0.03 \\
\hline & Pu-242 & 0.001 & 0.0039 & 0.00 \\
\hline & $\mathrm{Am}-241$ & 0 & 3.24 & 0.00 \\
\hline & & & & $9.75 \mathrm{E}-02$ \\
\hline \multirow[t]{7}{*}{$24 \% \mathrm{Pu}-240$} & Isotope & wt fraction & $\begin{array}{c}\text { Isotope } \\
\mathrm{Ci} / \mathrm{g}\end{array}$ & $\begin{array}{c}\text { Mixture } \\
\mathrm{Ci} / \mathrm{g}\end{array}$ \\
\hline & Pu-238 & 0.018 & 17.1 & 0.31 \\
\hline & Pu-239 & 0.542 & 0.062 & 0.03 \\
\hline & Pu-240 & 0.238 & 0.226 & 0.05 \\
\hline & Pu-242 & 0.064 & 0.0039 & 0.00 \\
\hline & Am-241 & 0 & 3.24 & 0.00 \\
\hline & & & & $3.95 \mathrm{E}-01$ \\
\hline \multirow[t]{6}{*}{ Depleted U } & Isotope & wt. Fraction & $\begin{array}{r}\text { Isotope } \\
\mathrm{Ci} / \mathrm{g}\end{array}$ & $\begin{array}{r}\text { Mixture } \\
\mathrm{Ci} / \mathrm{g}\end{array}$ \\
\hline & $\mathrm{U}-234$ & $4.2 \mathrm{E}-06$ & $6.20 \mathrm{E}-03$ & $2.60 \mathrm{E}-08$ \\
\hline & $\mathrm{U}-235$ & 0.0045 & $2.10 \mathrm{E}-06$ & $9.45 \mathrm{E}-09$ \\
\hline & $\mathrm{U}-238$ & $9.95 \mathrm{E}-01$ & $3.30 \mathrm{E}-07$ & $3.29 \mathrm{E}-07$ \\
\hline & & & & $3.64 \mathrm{E}-07$ \\
\hline & & & & \\
\hline
\end{tabular}




\begin{tabular}{|c|c|c|c|c|}
\hline \multirow[t]{5}{*}{ Natural U } & Isotope & wt. Fraction & $\begin{array}{r}\text { Isotope } \\
\mathrm{Ci} / \mathrm{g}\end{array}$ & $\begin{array}{r}\text { Mixture } \\
\mathrm{Ci} / \mathrm{g}\end{array}$ \\
\hline & U-234 & $5.5 \mathrm{E}-05$ & $6.20 \mathrm{E}-03$ & $3.41 \mathrm{E}-07$ \\
\hline & U-235 & 0.0072 & $2.10 \mathrm{E}-06$ & $1.51 \mathrm{E}-08$ \\
\hline & U-238 & $9.93 \mathrm{E}-01$ & $3.30 \mathrm{E}-07$ & $3.28 \mathrm{E}-07$ \\
\hline & & & & $6.84 \mathrm{E}-07$ \\
\hline \multirow{6}{*}{ Enriched U $(<20 \%)$} & & & & \\
\hline & Isotope & wt. Fraction & $\begin{array}{r}\text { Isotope } \\
\mathrm{Ci} / \mathrm{g}\end{array}$ & $\begin{array}{r}\text { Mixture } \\
\mathrm{Ci} / \mathrm{g}\end{array}$ \\
\hline & $\mathrm{U}-234$ & 0.0014 & $6.20 \mathrm{E}-03$ & $8.68 \mathrm{E}-06$ \\
\hline & U-235 & 0.2 & $2.10 \mathrm{E}-06$ & $4.20 \mathrm{E}-07$ \\
\hline & U-238 & 0.7986 & $3.30 \mathrm{E}-07$ & $2.64 \mathrm{E}-07$ \\
\hline & & & & $9.36 \mathrm{E}-06$ \\
\hline \multirow[t]{5}{*}{ Enriched U $(<90 \%)$} & Isotope & wt. Fraction & $\begin{array}{r}\text { Isotope } \\
\mathrm{Ci} / \mathrm{g}\end{array}$ & $\begin{array}{r}\text { Mixture } \\
\mathrm{Ci} / \mathrm{g}\end{array}$ \\
\hline & $\mathrm{U}-234$ & 0.0097 & $6.20 \mathrm{E}-03$ & $6.01 \mathrm{E}-05$ \\
\hline & $\mathrm{U}-235$ & 0.9 & $2.10 \mathrm{E}-06$ & $1.89 \mathrm{E}-06$ \\
\hline & $\mathrm{U}-238$ & 0.0903 & $3.30 \mathrm{E}-07$ & $2.98 \mathrm{E}-08$ \\
\hline & & & & $6.21 \mathrm{E}-05$ \\
\hline
\end{tabular}

Reference for Pu and U mixes isotope weight percent: Sula, M.J., E.H. Carbaugh, and D.E. Bihl. 1991. Technical Basis for Internal Dosimetry at Hanford, PNL-6866, Rev. 1, Pacific Northwest Laboratory, Richland, Washington. 


\section{Appendix B}

\section{Radionuclide Inventory Database Features}

The information obtained from the nuclear materials inventory, composite radioactive materials inventory, and facility management radioactive materials inventory is maintained by Effluent Management (EM) in an ACCESS database. The inventory information that is obtained and stored in the database is listed in Section 2.1.

\section{Database Population}

The nuclear materials and composite radioactive materials inventories currently are obtained in electronic formats. The general format of the electronic files is manipulated to allow electronic downloading of the information from these two sources into the database tables, the data in the electronic files is not changed.

The facility management radioactive materials inventory is obtained from individual research personnel who act as custodians of the material. This inventory data is provided in various forms (handwritten, electronic files, e-mail messages, etc.) Electronic files are reformatted to the database table format and downloaded directly into the database tables. Handwritten and e-mail information is manually input to the database tables by EM personnel. Entries to the database are independently verified by other EM personnel for accuracy.

The information entered into the database includes:

- Staff member acting as custodian of the material

- Research or support division of the custodian

- Form of the material - gas, liquid, powder, solid, contained, or exempt/sealed DOT

- Basis for the Inventory - physical inventory records, estimated through process knowledge, estimated through transportation records, or estimated through other means (i.e., procedures, documents, etc.)

- Nuclide

- Inventory in activity (Ci) or mass (grams)

- Building/Room in which material is stored or used

- Identification number - numbers previously assigned by the custodian or by other databases (sealed source or material balance area identification numbers)

- Comments - any additional comments related to the material (e.g., reference numbers on the material, whether or not the material is considered throughput, description of the material, etc.).

The database assigns an identification number to each of the entries. For sealed source and Material Balance Area (MBA) data the identification number previously assigned to the material is used. For the research inventories, the database applies a numerical sequential number to each 
of the entries. The database assigns the following designations to the identification number:

D - = Input provided by the research division

$\mathrm{G}-=$ Government sealed source

$\mathrm{P}$ - = Private radioactive material inventory

$\mathrm{M}-=$ Nuclear materials inventory.

\section{Other Database Tables}

Other database tables are used to store specific information about individual nuclides that are used in the NESHAP dose calculations:

Factors Table - Factors for the dose per curie $(\mathrm{mrem} / \mathrm{Ci})$ for different isotopes are listed in Table 3 and are entered as a separate table in the ACCESS database. The source for each of the nuclide factors is listed in the factors table.

Release Fraction Table - The release fractions for material forms (i.e. solid, liquid, powder, etc.) are listed in Table 2. These are entered as a separate table in the database.

\section{Calculations}

The ACCESS database uses queries and macros that are applied to the tables listed above to calculate the potential dose for the different Pacific Northwest facilities.

Normalizing Inventory Data -- The database is designed to convert the reported mass and activity inventory units (i.e., grams, $\mathrm{mCi}, \mu \mathrm{Ci}, \mathrm{mg}, \mu \mathrm{g}$, etc.) to curie (Ci) units for use in subsequent calculations.

Potential Dose Calculations -- Potential dose calculations are determined on a facility-specific basis. The reported inventory is first converted to $\mathrm{Ci}$ and then it is multiplied by the dose factor $(\mathrm{mrem} / \mathrm{Ci})$ for the specific nuclide, the location modification factor, and the release fraction to determine the potential dose for that nuclide inventory.

Example: $\quad 20 \mu \mathrm{Ci}$ of $\mathrm{U}-238$ in powder form at the 305B facility $20 \mu \mathrm{Ci} * 1.0 \mathrm{E}-06 \mathrm{Ci} / \mu \mathrm{Ci} * 1 \mathrm{E}-03 * 6.2 \mathrm{E}+01 \mathrm{mrem} / \mathrm{Ci} * 1.0=1.24 \mathrm{E}-06 \mathrm{mrem} / \mathrm{Ci}$

Where : $\quad 1 \mathrm{E}-03=$ release fraction for powder $6.2 \mathrm{E}+01 \mathrm{mrem} / \mathrm{Ci}=$ dose factor for $\mathrm{U}-238$

$1.0=$ location modification factor (with relation to 300 Area)

The cumulative dose for the facility is determined by summing the potential doses of each inventory entry. 


\section{Reports}

Three reports are generated using the database for the NESHAP assessment as part of the final packet:

- The first is a report of the "raw" data that was provided by the research personnel, the nuclear materials inventory, and the composite radioactive materials inventory.

- The second is a summary page listing the potential dose ( $\mathrm{mrem} / \mathrm{Ci}$ ) of the facility inventory and sign-off blocks for the preparer, reviewer, facility safety representative, divisional representative(s), and the building manager.

- The third is a listing of each radionuclide present in the building and the dose contribution in $\mathrm{mrem} / \mathrm{Ci}$ and in $\%$ of the total dose. 
Appendix C

Unit Dose Factor Calculations 
Project No.

Date June 3,1996
To MY Ballinger
From LH Staven
Subject 331 Building Unit Ci Release Dose Calculations

MJ Sula

K Rhoads

File/LB

Please find attached the Unit Ci dose calculations for the 331 Building. These calculations have been reviewed by Kathy Rhoads for HEDOP approval. The 331 values are reported with the 9 year meteorological data.

These values were calculated based on the EMSL values reported earlier, and are adjusted by 331 specific $X / Q$ values. During the preparation of these calculations, USGS quadrangle maps were made available for determining distances to the maximally exposed individuals. These maps will assure consistency in reporting dose conversion values. Please use these values for your reporting purposes for the 331 Building.

If you have any questions, please contact me at (509) 375-2429.

Attachment 


\section{HEDOP REVIEW CHECKLIST \\ for \\ Radiological and Nonradiological Release Calculations}

Document reviewed (include title or description of calculation, document number, author, and date, as applicable):

CAP88-PC Dose Conversion Factors for 331 Building 5/22/96

Submitted by:

L. H. Staven

Date Submitted: $5 / 22 / 96$

Scope of Review:

YES NO* N/A

[x] [ ] [ ] 1. A detailed technical review and approval of the environmental transport and dose calculation portion of the analys is has been performed and documented.

[x] [ ] [ ] 2. Detailed technical review(s) and approval(s) of scenario and release determinations have been performed and documented.

[x] [ ] [ ] 3. HEDOP-approved code(s) were used.

$[x][] \quad[$ ] 4. Receptor locations were selected according to HEDOP recommendations.

[x] [ ] [ ] 5. All applicable environmental pathways and code options were included and are appropriate for the calculations.

[x] [ ] [ ] 6. Hanford site data were used.

$[x]\left[\begin{array}{l}{[} \\ {[}\end{array}\right]$ 7. Model adjustments external to the computer program were justified and performed correctly.

[x] [ ] [ ] 8. The analys is is consistent with HEDOP recommendations.

9. Supporting notes, calculations, comments, comment resolutions, or other information is attached. (Use the "Page 1 of $X$ " page numbering format and sign and date each added page.)

[x] [ ]

10. Approval is granted on behalf of the Hanford Environmental Dose Overview Panel.

* A11 "NO" responses must be explained and use of nonstandard methods justified.

$\frac{\text { K. Rhoads }}{\text { HEDOP-Approved Reviewer (Printed Name and Signature) }}$

COMMENTS (add additional signed and dated pages if necessary): 


\section{APPLICATION REPORT}

1) Project title and number CAP88-PC DOSE CONVERSION FACTORS FOR 331 BUILDING 5/22/96

2) Purpose of application package and relationship to other work:

This series of CAP88-PC runs were produced in order to report the 50-year total effective dose equivalent (TEDE) to the maximally exposed individual (MEI) and offsite worker per $\mathrm{Ci}$ released from the 331 Building, Table 1. These values will be used to demonstrate NESHAPs (National Emissions Standards for Hazardous Air Pollutants) compliance.

This application is based on the work performed in January ' 96 for the EMSL facility. The dose factors for the EMSL facility were multiplied by the appropriate conversion factor. Each Conversion factor is specific to the location of the MEI of interest, and is determined by the ratio of the (331 MEl/EMSL MEI) chi/Q values.

3) List original sources of input data, assumptions and derivations used to obtain it, and justification for its use, as appropriate. (If input information has been previously reviewed, reference the documentation of this review.)

Dose factors were created for both the offsite MEI and the onsite MEI.

Worker doses were calculated based on $100 \%$ import of food products. To adjust for 2000 hours per year at the site, the CAP 88 PC dose was adjusted by $2000 / 8766$.

331 Building parameters included a $10 \mathrm{~m}$ release height.

Hanford parameters listed in Schreckhise et al (1993) were used in place of default CAP88-PC default parameters to better model the Hanford environment.

4) Minor changes made in the software that produced the application run (see section 4.1). N/A

5) Describe interrelationships and dependencies of each application run in the application package.

The TEDEs for the CAP88-PC radionuclides were adjusted to represent the value with the contribution from progeny which were grown-in after the release from the facility. The adjustment factors were derived from previous GENII runs (FEMP 3/93) where only the parent is released, and the output includes the doses from the progeny. The adjustment factor was taken by dividing the dose from the parent by the total dose from the parent and progeny. The CAP88-PC value was then divided by the factor to represent the total dose. Where CAP88-PC 
calculated chains (i.e., Cs-137) the progeny were added to the parent, and no other adjustment was made.

6) Summarize the overall output of the application package in relation of the purpose stated in item 2 above (including tables and graphs, as appropriate):

Table 1 shows the worker and resident 50 year TEDE conversion factors per $\mathrm{Ci}$ released.

7) Submitted for ITR by:

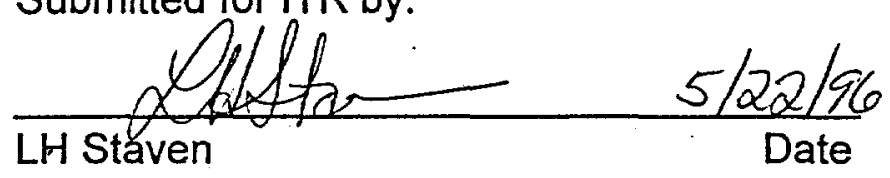

8) Approved for reporting results to sponsor by:

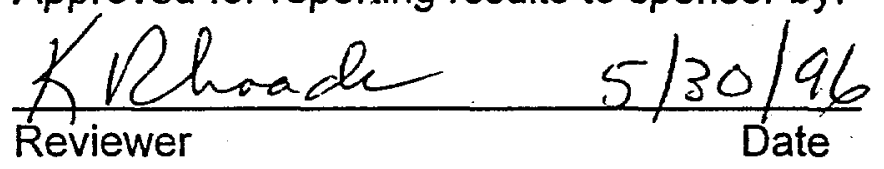

C.5 


\section{APPLICATION RECORD LOG}

1) Project title and number: CAP88-PC Dose Conversion Factors for EMSL Building

2)Application 3) Date and Time 4)Software Name (title) 5) computer type Record Log of Run

Number

Filename.(a) see below operating system

compilers/libraries and Version
6) Comments Identifier
7)Data input

CAP88-PC Ver $1.0 \quad$ Gateway2000 486

see below

see below

\section{Directory of D: \CAP8BPC\OUTPUT}

Directory of D:ICAP88PCIOUTPUT

Worker

331rn wrkr onsit

331rn wrkr offsi

C. . SUM

$9635 \quad 05 / 22 / 96 \quad 14: 33$

Resident

D. . SUM $9635 \quad 05 / 22 / 96 \quad 14: 27$

331 rn resid

B $\quad$.SUM

$963505 / 07 / 96 \quad 12: 21$

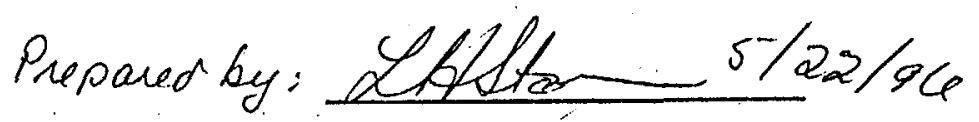




\begin{tabular}{|c|c|c|c|c|c|c|c|c|}
\hline \multicolumn{7}{|c|}{ Table 1. CAP88PC Dose Factors for Unit Ci Releases From 331 Building } & \\
\hline & & & & & & & $\frac{1}{16}$ & \\
\hline & & fraction of & Offsite & & Onsite & & & \\
\hline & & dose from & Worker & & Worker & & Residential & \\
\hline & $X / Q=$ & parent & $2.82 \mathrm{E}-06$ & & $1.49 \mathrm{E}-05$ & & $1.77 \mathrm{E}-06$ & \\
\hline & Location & & $1000 \mathrm{~m} \mathrm{NY}$ & & $200 \mathrm{~m} \mathrm{~S}$ & & $1000 \mathrm{mE}$ & \\
\hline & Duration & & $2000 \mathrm{~h} / \mathrm{y}$ & & $2000 \mathrm{~h} / \mathrm{y}$ & & $8766 \mathrm{~h} / \mathrm{y}$ & \\
\hline \multicolumn{3}{|c|}{ Food locally produced } & $0 \%$ & & $0 \%$ & & $100 \%$ & \\
\hline & & & mrem/yr & & mrem/yr & & mrem/yr & \\
\hline & \multicolumn{2}{|c|}{ Radionuclide } & \multicolumn{2}{|c|}{ per Ci released } & \multicolumn{2}{|c|}{ per Ci released } & \multicolumn{2}{|c|}{ per Ci released } \\
\hline & & & & & & & & \\
\hline & $\mathrm{H}-3$ & & 2.3E-05 & & $1.22 \mathrm{E}-04$ & & 4.1E-04 & \\
\hline & $\mathrm{BE}-7$ & & $1.3 \mathrm{E}-03$ & & $6.87 E-03$ & & $4.5 \mathrm{E}-03$ & \\
\hline & C-11 & & $7.2 E-05$ & & $3.80 E-04$ & & $1.9 \mathrm{E}-04$ & \\
\hline & C-14 & & 2.1E-06 & & $1.12 \mathrm{E}-05$ & & $5.0 \mathrm{E}-02$ & \\
\hline & $\mathrm{N}-13$ & & 4.8E-05 & & $2.55 E-04$ & & $1.3 \mathrm{E}-04$ & \\
\hline & $0-15$ & & $5.6 \mathrm{E}-06$ & & $2.96 \mathrm{E}-05$ & & $1.5 \mathrm{E}-05$ & \\
\hline & $F-18$ & & $1.3 \mathrm{E}-04$ & & $7.08 \mathrm{E}-04$ & & $3.6 E-04$ & \\
\hline & NA-22 & & $8.4 \mathrm{E}-01$ & & $4.45 E+00$ & & $2.4 \mathrm{E}+00$ & \\
\hline & NA-24 & & 1.7E-03 & & $8.80 \mathrm{E}-03$ & & $4.5 \mathrm{E}-03$ & \\
\hline & P-32 & & $1.0 \mathrm{E}-03$ & & $5.31 \mathrm{E}-03$ & & $5.7 \mathrm{E}-02$ & \\
\hline & S-35 & & $4.9 \mathrm{E}-05$ & & $2.61 \mathrm{E}-04$ & & $6.2 \mathrm{E}-03$ & \\
\hline & AR-41 & & $1.2 \mathrm{E}-04$ & & $6.49 E-04$ & & $3.3 \mathrm{E}-04$ & \\
\hline & $K-40$ & & $5.0 \mathrm{E}-01$ & & $2.64 \mathrm{E}+00$ & & $3.3 E+00$ & \\
\hline & CA-41 & & $7.5 \mathrm{E}-05$ & & $3.97 \mathrm{E}-04$ & & $2.0 E-04$ & \\
\hline & $S C-46$ & & $7.8 \mathrm{E}-02$ & & $4.14 \mathrm{E}-01$ & & $2.7 \mathrm{E}-01$ & \\
\hline & CR-51 & & $4.8 \mathrm{E}-04$ & & $2.55 \mathrm{E}-03$ & & $2.3 \mathrm{E}-03$ & \\
\hline & MN-54 & & $1.2 \mathrm{E}-01$ & & $6.21 \mathrm{E}-01$ & & $3.4 \mathrm{E}-01$ & \\
\hline & MN-56 & & $3.2 \mathrm{E}-04$ & & $1.72 \mathrm{E}-03$ & & $8.7 \mathrm{E}-04$ & \\
\hline & FE-55 & & $3.2 \mathrm{E}-04$ & & $1.67 \mathrm{E}-03$ & & $6.9 \mathrm{E}-03$ & \\
\hline & FE-59 & & $2.4 \mathrm{E}-02$ & & $1.29 \mathrm{E}-01$ & & $1.2 \mathrm{E}-01$ & \\
\hline & $C O-57$ & & $1.8 \mathrm{E}-02$ & & $9.70 \mathrm{E}-02$ & & $6.1 \mathrm{E}-02$ & \\
\hline & CO-58 & & $3.3 E-02$ & & $1.77 \mathrm{E}-01$ & & $1.2 \mathrm{E}-01$ & \\
\hline & CO-60 & & $1.8 \mathrm{E}+00$ & & $9.32 \mathrm{E}+00$ & & $5.0 E+00$ & \\
\hline & NI-59 & & $1.7 \mathrm{E}-03$ & & $8.84 \mathrm{E}-03$ & & $8.1 \mathrm{E}-03$ & \\
\hline & NII-63 & & $3.8 \mathrm{E}-04$ & & $2.01 \mathrm{E}-03$ & & $1.0 \mathrm{E}-02$ & \\
\hline & NI-65 & & $1.3 \mathrm{E}-04$ & & 6.87E-04 & & $3.5 \mathrm{E}-04$ & \\
\hline & CU-64 & & $1.2 \mathrm{E}-04$ & & $6.52 \mathrm{E}-04$ & & $3.4 \mathrm{E}-04$ & \\
\hline & $\mathrm{ZN}-65$ & & $6.3 \mathrm{E}-02$ & & $3.34 \mathrm{E}-01$ & & $4.3 \mathrm{E}-01$ & \\
\hline & ZN-69 & & $9.6 \mathrm{E}-06$ & & 5.07E-05 & & $2.6 \mathrm{E}-05$ & \\
\hline & $\mathrm{ZN}-69 \mathrm{M}$ & & $3.2 E-04$ & & $1.70 \mathrm{E}-03$ & & $8.8 \mathrm{E}-04$ & \\
\hline & GA-67 & & $3.5 E-04$ & & $1.86 \mathrm{E}-03$ & & $1.7 \mathrm{E}-03$ & \\
\hline & AS-76 & & $1.0 \mathrm{E}-03$ & & $5.28 \mathrm{E}-03$ & & $3.0 \mathrm{E}-03$ & \\
\hline & BR-82 & & $2.2 \mathrm{E}-03$ & & $1.16 \mathrm{E}-02$ & & $6.3 \mathrm{E}-03$ & \\
\hline & BR-83 & & $1.0 \mathrm{E}-06$ & & $5.28 \mathrm{E}-06$ & & $2.7 \mathrm{E}-06$ & \\
\hline & BR-84 & & $1.6 \mathrm{E}-04$ & & $8.49 \mathrm{E}-04$ & & $4.3 \mathrm{E}-04$ & \\
\hline & BR-85 & & $7.9 E-07$ & & $4.18 E-06$ & & $2.1 \mathrm{E}-06$ & \\
\hline & KR-83M & & $2.2 \mathrm{E}-08$ & & 1.17E-07 & & $6.0 \mathrm{E}-08$ & \\
\hline & KR-85 & & 3.4E-07 & - & 1.81E-06 & & $9.2 \mathrm{E}-07$ & \\
\hline & KR-85M & & $1.6 \mathrm{E}-05$ & & $8.35 \mathrm{E}-05$ & & $4.3 E-05$ & \\
\hline & KR-87 & & $8.0 \mathrm{E}-05$ & & $4.21 E-04$ & & $2.1 \mathrm{E}-04$ & \\
\hline & KR-88 & & $2.2 \mathrm{E}-04$ & & $1.15 \mathrm{E}-03$ & & $5.9 \mathrm{E}-04$ & \\
\hline & KR-89 & & $2.8 \mathrm{E}-05$ & & $1.50 \mathrm{E}-04$ & & $7.6 \mathrm{E}-05$ & \\
\hline & KR-90 & & $1.3 \mathrm{E}-08$ & & $6.94 \mathrm{E}-08$ & & $3.5 \mathrm{E}-08$ & \\
\hline & RB-86 & & $2.1 \mathrm{E}-03$ & & $1.13 \mathrm{E}-02$ & & $7.2 \mathrm{E}-02$ & \\
\hline & RB-87 & & $1.2 \mathrm{E}-03$ & & $6.49 \mathrm{E}-03$ & & $2.6 \mathrm{E}-01$ & \\
\hline & RB-88 & & $5.7 \mathrm{E}-05$ & & $3.01 \mathrm{E}-04$ & & $1.5 \mathrm{E}-04$ & \\
\hline & RB-89 & & $1.4 \mathrm{E}-04$ & & $7.35 \mathrm{E}-04$ & & $3.7 \mathrm{E}-04$ & \\
\hline & RB-90 & & $2.4 E-05$ & & $1.29 \mathrm{E}-04$ & & $6.6 \mathrm{E}-05$ & \\
\hline & RB-90M & & $7.8 \mathrm{E}-05$ & & $4.14 \mathrm{E}-04$ & & $2.1 \mathrm{E}-04$ & \\
\hline
\end{tabular}

- Includes dose from long-lived progeny

* Includes dose from short-lived progeny 


\begin{tabular}{|c|c|c|c|c|c|c|c|c|}
\hline \multicolumn{9}{|c|}{ Table 1. CAP88PC Dose Factors for Unit Ci Releases From 331 Building } \\
\hline & & & & & & & & \\
\hline & & & & & & & & \\
\hline & & fraction of & Offsite & & Onsite & & & \\
\hline & & dose from & Worker & & Worker & & Residential & \\
\hline & $X / Q=$ & parent & \begin{tabular}{|l|}
$2.82 \mathrm{E}-06$ \\
\end{tabular} & & $1.49 \mathrm{E}-05$ & & $1.77 \mathrm{E}-06$ & \\
\hline & Location & & $1000 \mathrm{~m} \mathrm{NW}$ & & $200 \mathrm{~m} \mathrm{~S}$ & & $1000 \mathrm{mE}$ & \\
\hline & Duration & & $2000 \mathrm{~h} / \mathrm{y}$ & & $2000 \mathrm{~h} / \mathrm{y}$ & & $8766 \mathrm{~h} / \mathrm{y}$ & \\
\hline \multicolumn{3}{|c|}{ Food locally produced } & $0 \%$ & & $0 \%$ & & $100 \%$ & \\
\hline & & & mrem/yr & & $\mathrm{mrem} / \mathrm{yr}$ & & mrem/yr & \\
\hline & \multicolumn{2}{|c|}{ Radionuclide } & \multicolumn{2}{|c|}{ per Ci released } & \multicolumn{2}{|c|}{ per Ci released } & \multicolumn{2}{|c|}{ per Ci released } \\
\hline & & & & & & & & \\
\hline & SR-89 & & $1.1 \mathrm{E}-03$ & & $5.73 E-03$ & & $7.6 \mathrm{E}-02$ & \\
\hline & SR-90* & 0.94 & $3.9 \mathrm{E}-02$ & & $2.07 E-01$ & & $4.9 E+00$ & \\
\hline- & SR-91 & & \begin{tabular}{|c|}
$3.7 E-04$ \\
\end{tabular} & & $1.94 \mathrm{E}-03$ & & $1.0 \mathrm{E}-03$ & \\
\hline & SR-92 & & $2.9 \mathrm{E}-04$ & & $1.53 \mathrm{E}-03$ & & $7.8 \mathrm{E}-04$ & \\
\hline & $Y-90$ & & $1.6 \mathrm{E}-03$ & & $8.25 \mathrm{E}-03$ & & 1.1E-02 & - \\
\hline & Y-90M & & 9.7E-05 & & 5.11E-04 & & $2.6 \mathrm{E}-04$ & \\
\hline & $\overline{Y-91}$ & & $8.2 \mathrm{E}-03$ & & $4.35 \mathrm{E}-02$ & & $9.8 \mathrm{E}-02$ & \\
\hline & $Y-91 M$ & & 5.6E-05 & & $2.94 \mathrm{E}-04$ & & $1.5 \mathrm{E}-04$ & \\
\hline & $\overline{Y-92}$ & & $2.3 E-04$ & & $1.23 \mathrm{E}-03$ & & $6.3 \mathrm{E}-04$ & \\
\hline & Y-93 & & $4.9 \mathrm{E}-04$ & & $2.58 \mathrm{E}-03$ & & $1.3 \mathrm{E}-03$ & \\
\hline & ZR-93 & & $8.0 \mathrm{E}-03$ & & $4.21 \mathrm{E}-02$ & & $3.2 \mathrm{E}-02$ & \\
\hline & ZR-95* & 0.75 & $3.2 \mathrm{E}-02$ & & $1.69 \mathrm{E}-01$ & & $1.3 \mathrm{E}-01$ & \\
\hline & NB-93M & & $6.5 \mathrm{E}-03$ & & $3.45 \mathrm{E}-02$ & & $2.8 \mathrm{E}-02$ & \\
\hline & NB-94 & & $5.8 \mathrm{E}+00$ & & $3.09 E+01$ & & $1.6 E+01$ & \\
\hline & NB-95 & & $1.3 \mathrm{E}-02$ & & $7.08 E-02$ & & $6.4 \mathrm{E}-02$ & \\
\hline & NB-95M & & $5.3 \mathrm{E}-04$ & & $2.80 \mathrm{E}-03$ & & $4.6 \mathrm{E}-03$ & \\
\hline & NB-97 & & $9.2 \mathrm{E}-05$ & & $4.87 \mathrm{E}-04$ & & $2.5 \mathrm{E}-04$ & \\
\hline . & NB-97M & & $3.5 \mathrm{E}-07$ & & $1.84 \mathrm{E}-06$ & & $9.4 \mathrm{E}-07$ & \\
\hline & MO-93 & & $2.1 \mathrm{E}-02$ & & $1.10 E-01$ & & $5.6 \mathrm{E}-02$ & \\
\hline & MO-99** & & $9.2 \mathrm{E}-03$ & & $6.12 \mathrm{E}-03$ & & 5.2E-03 & \\
\hline & TC-99M & & $0.0 E+00$ & & $0.00 \mathrm{E}+00$ & & $0.0 E+00$ & \\
\hline & TC-97 & & $2.3 \mathrm{E}-02$ & & $1.23 \mathrm{E}-01$ & & $1.3 \mathrm{E}-01$ & \\
\hline & TC-99 & & $1.4 \mathrm{E}-03$ & & $7.39 \mathrm{E}-03$ & & $6.2 \mathrm{E}-01$ & \\
\hline & TC-101 & & $1.9 E-05$ & & $1.03 E-04$ & & $5.2 \mathrm{E}-05$ & \\
\hline & RU-97 & & $4.3 E-04$ & & $2.26 \mathrm{E}-03$ & & $1.6 \mathrm{E}-03$ & \\
\hline & RU-103 & & $1.0 \mathrm{E}-02$ & & $5.52 \mathrm{E}-02$ & & $5.0 \mathrm{E}-02$ & \\
\hline & $R U-105$ & & $2.4 E-04$ & & $1.29 \mathrm{E}-03$ & & $6.6 \mathrm{E}-04$ & \\
\hline & RU-106 & & $7.9 E-02$ & & $4.18 \mathrm{E}-01$ & & 4.7E-01 & \\
\hline & RH-105 & & $2.4 E-04$ & & $1.29 \mathrm{E}-03$ & & $9.4 \mathrm{E}-04$ & \\
\hline & $\overline{R H}-105 \mathrm{M}$ & & $2.9 \mathrm{E}-09$ & & $1.54 \mathrm{E}-08$ & & $7.8 \mathrm{E}-09$ & \\
\hline & RH-106 & & $1.0 \mathrm{E}-09$ & & $5.45 E-09$ & & $2.8 \mathrm{E}-09$ & \\
\hline & PD-107 & & $2.1 E-03$ & & $1.11 \mathrm{E}-02$ & & $8.5 E-03$ & \\
\hline & PD-109 & & 2.7E-04 & & 1.43E-03 & & $7.6 \mathrm{E}-04$ & \\
\hline & AG-109M & & $2.1 \mathrm{E}-10$ & & $1.09 \mathrm{E}-09$ & & $5.5 \mathrm{E}-10$ & \\
\hline & AG-110 & & $2.4 \mathrm{E}-11$ & & $1.25 \mathrm{E}-10$ & & $6.4 \mathrm{E}-11$ & \\
\hline & AG-110M & & $3.1 \mathrm{E}-01$ & & $1.65 E+00$ & & $9.6 \mathrm{E}-01$ & \\
\hline & AG-111 & & $1.2 \mathrm{E}-03$ & & $6.28 E-03$ & & $1.9 \mathrm{E}-02$ & \\
\hline & CD-115 & & $1.0 \mathrm{E}-03$ & & 5.31E-03 & & $5.2 \mathrm{E}-03$ & \\
\hline & CD-115M & & $7.7 \mathrm{E}-03$ & & $4.07 E-02$ & & $1.4 \mathrm{E}-01$ & \\
\hline & $\mid N-113 M$ & & $3.8 \mathrm{E}-05$ & & $2.03 E-04$ & & $1.0 E-04$ & \\
\hline & $\mathbb{I N - 1 1 5}$ & & $1.7 \mathrm{E}-01$ & & $9.01 \mathrm{E}-01$ & & $2.0 E+00$ & \\
\hline & IN-115M & & $5.7 E-05$ & & $3.02 E-04$ & & $1.5 \mathrm{E}-04$ & \\
\hline & $\mathrm{SN}-113$ & & $2.6 \mathrm{E}-03$ & & $1.39 \mathrm{E}-02$ & & $4.0 \mathrm{E}-02$ & \\
\hline & $S N-123$ & & $3.8 \mathrm{E}-04$ & & $2.01 \mathrm{E}-03$ & & $1.0 \mathrm{E}-03$ & \\
\hline & SN-125 & & $4.0 \mathrm{E}-03$ & & $2.11 \mathrm{E}-02$ & & $5.7 \mathrm{E}-02$ & \\
\hline & SN-126 & & $2.3 \mathrm{E}-01$ & & $1.23 E+00$ & & $9.0 \mathrm{E}-01$ & \\
\hline & $\widehat{S B-124}$ & & $5.2 E-02$ & & $2.77 \mathrm{E}-01$ & & $2.2 \mathrm{E}-01$ & \\
\hline & SB-125 & & $1.9 \mathrm{E}-01$ & & $1.00 \mathrm{E}+00$ & & $5.4 \mathrm{E}-01$ & \\
\hline & SB-126 & & $1.8 \mathrm{E}-02$ & & $9.56 \mathrm{E}-02$ & & $9.1 E-02$ & \\
\hline
\end{tabular}




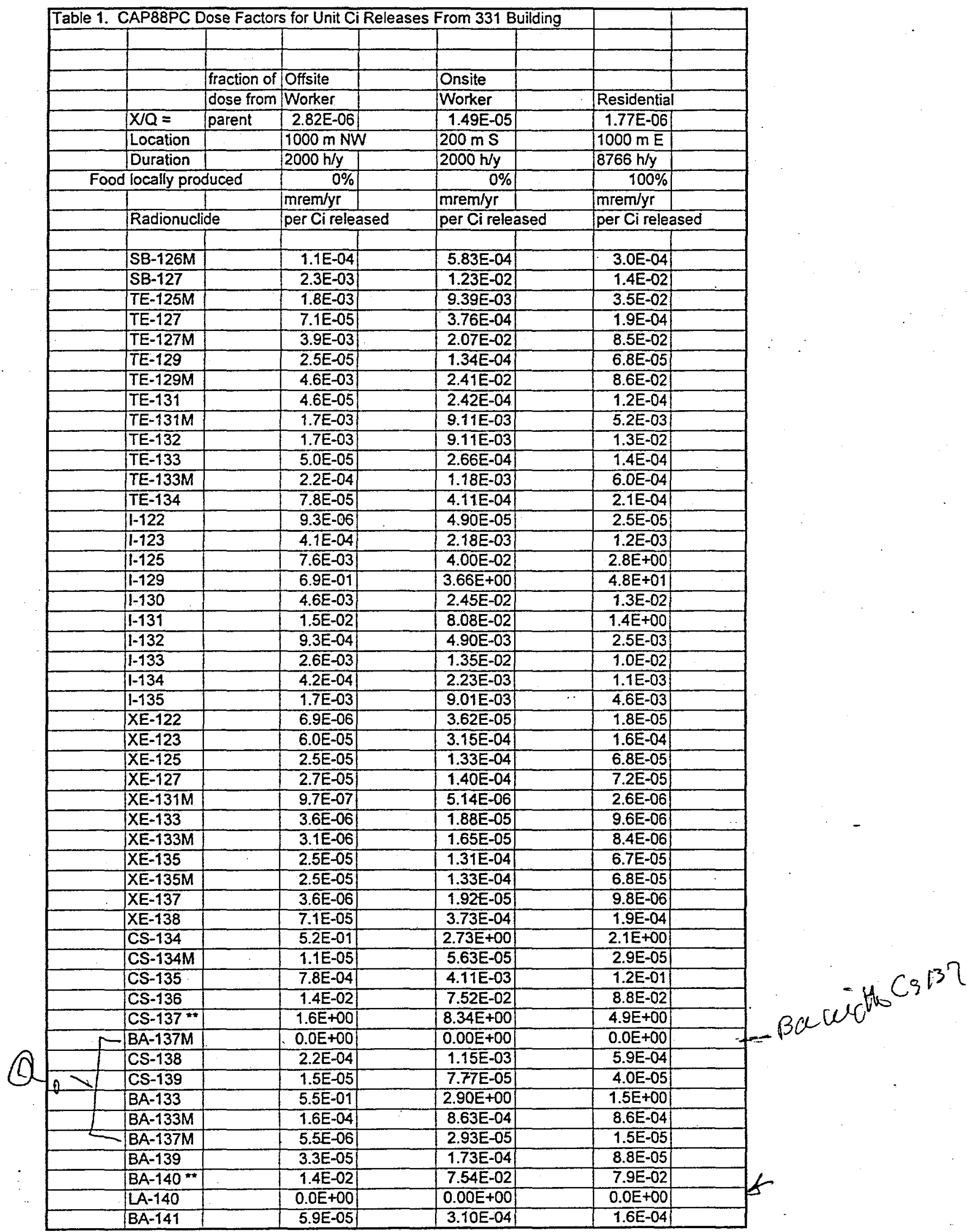




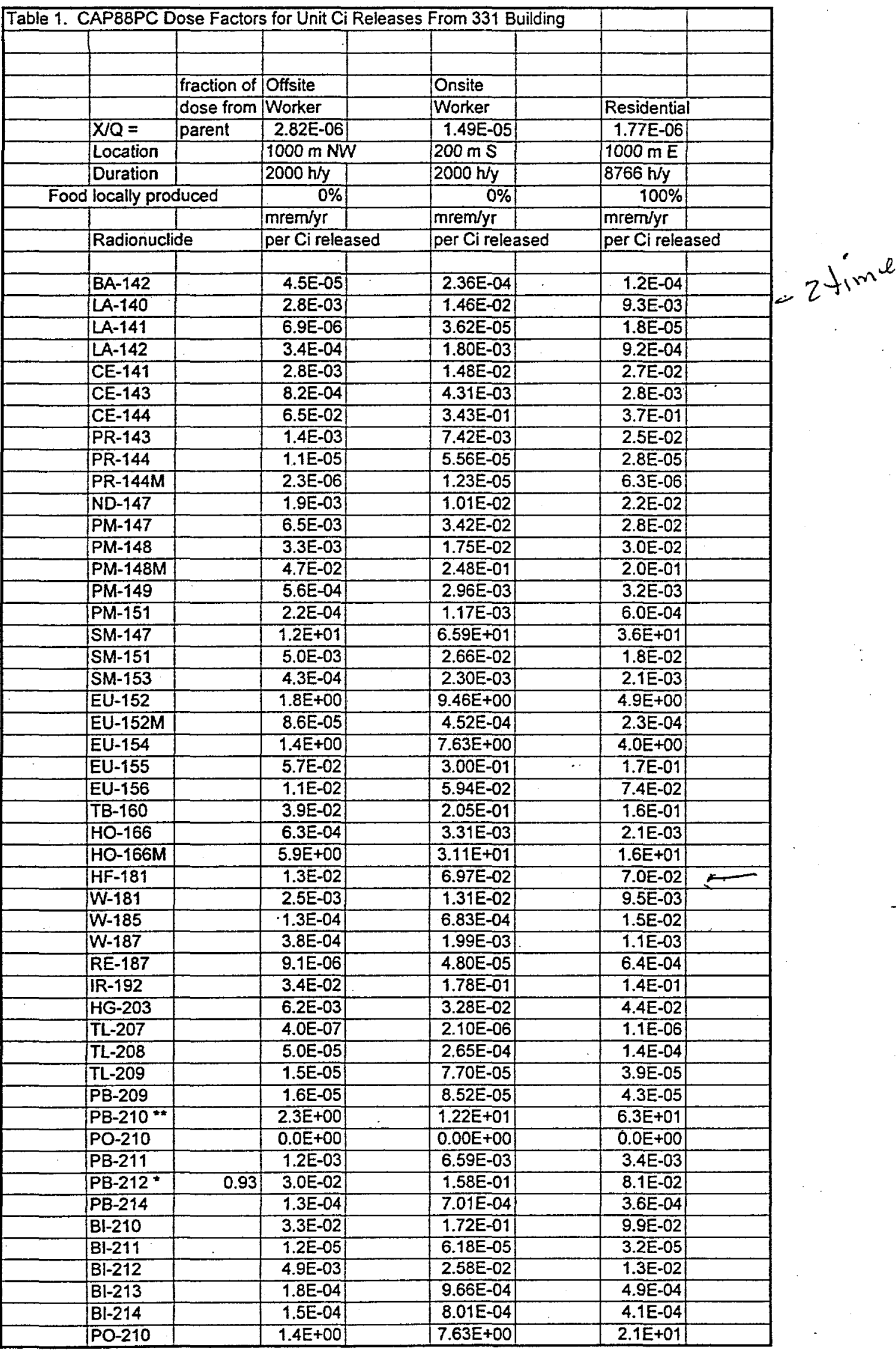




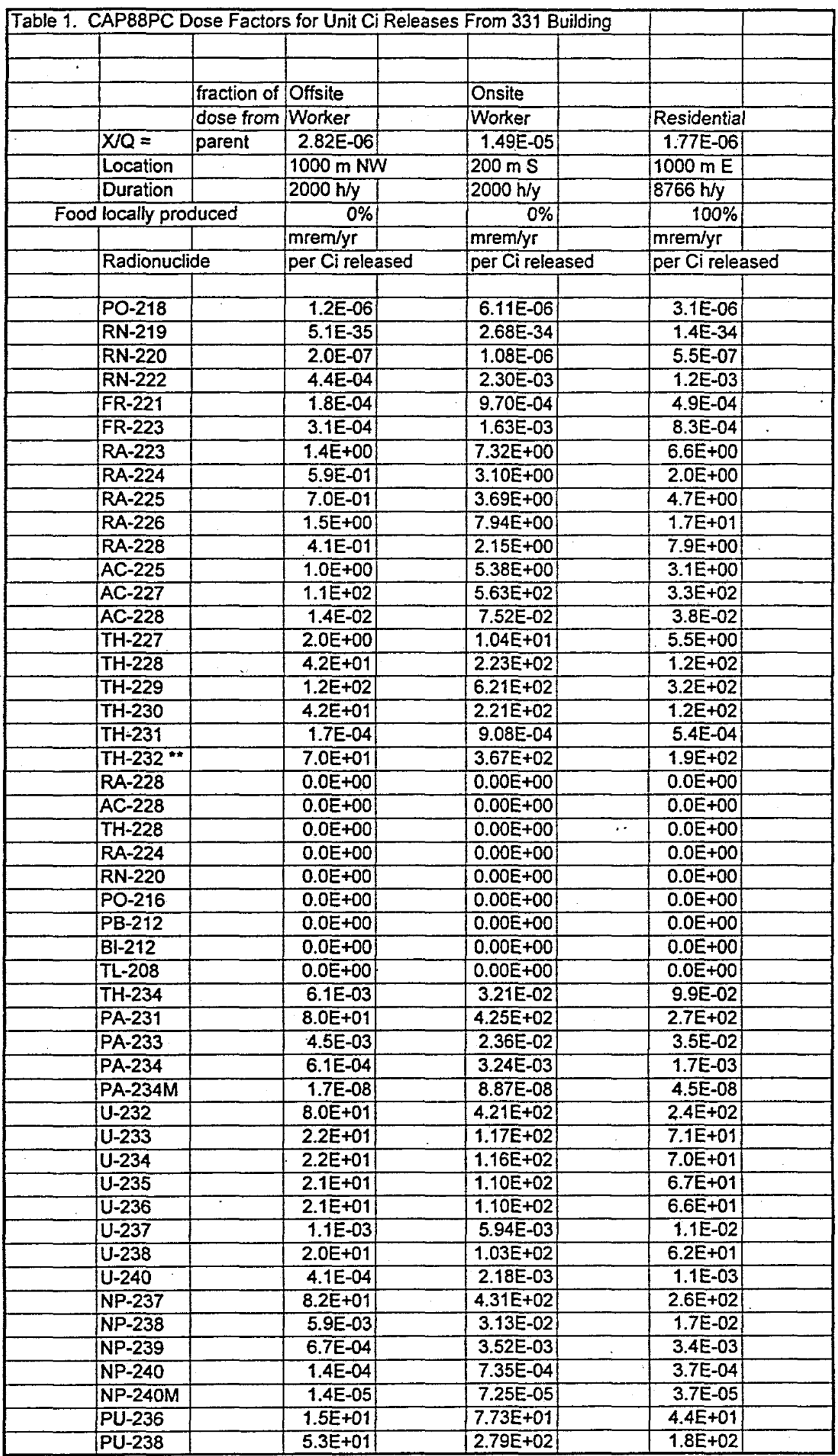

* Includes dose from long-lived progeny

* Includes dose from short-lived progeny 


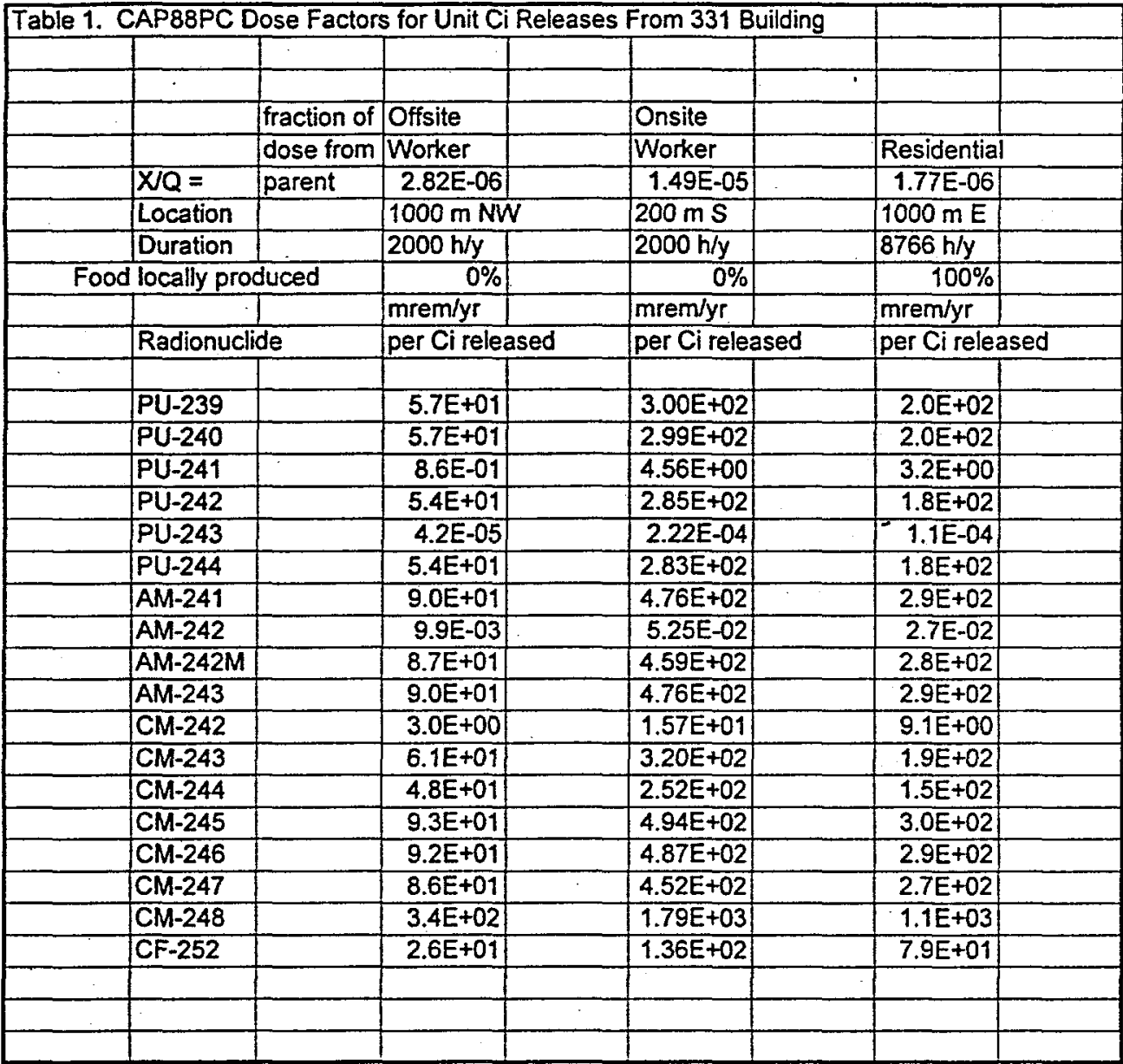




\section{Distribution}

No. of

Copies

OFFSITE

1 DOE/Office of Scientific and Technical Information

ONSITE

2 DOE Richland Operations Office

M. E. Burandt

K8-50

S. D. Stites

R3-79
No. of

Copies

20 Pacific Northwest National Laboratory

B. P. Atencio

P7-68

M. Y. Ballinger

BSRC

E. G. Damberg

P7-68

D. L. Edwards (5)

P7-68

T. M. Graham

P7-75

S. L. Jones

P7-08

A. S. Ikenberry

K. D. Shields

P7-79

P7-68

M. S. Sula

Clearance Office (7)

SEQUIM 